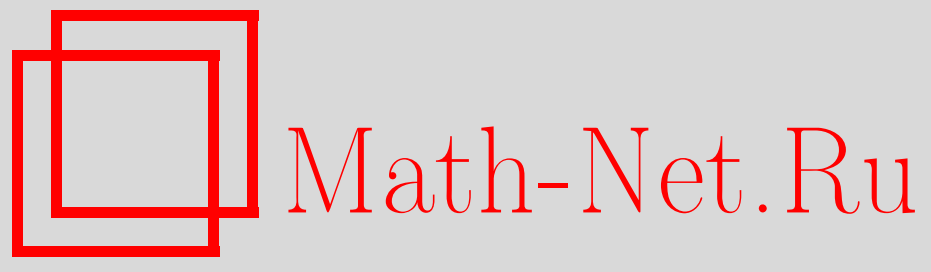

М. М. Гриненко, Расслоения на поверхности дель Пеццо, УМH, 2006, том 61, выпуск 2, 67-112

DOI: https://doi.org/10.4213/rm1722

Использование Общероссийского математического портала Math-Net.Ru подразумевает, что вы прочитали и согласны с пользовательским соглашением http://www . mathnet.ru/rus/agreement

Параметры загрузки:

IP: 18.209 .158 .208

26 апреля 2023 г., 15:40:27

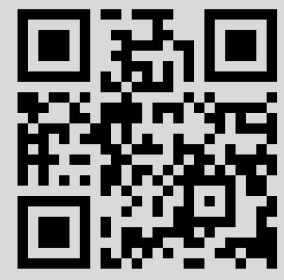


УДК 512.76

\section{Расслоения на поверхности дель Пеццо}

\section{М. М. Гриненко}

В статье дан обзор бирациональной геометрии трехмерных расслоений на поверхности дель Пеццо. Рассматриваются конструкции таких многообразий, проблемы их рациональности, бирациональной жесткости, смежные вопросы.

Библиография: 48 названий.

\section{СОДЕРЖАНИЕ}

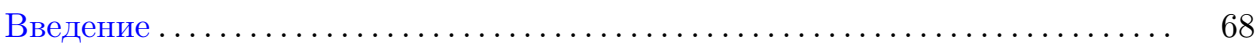

1. Структуры Мори .................................. 68

2. Программа Саркисова и метод максимальных особенностей......... 72

3. Поверхности дель Пеццо ................................... 74

3.1. Горенштейновы поверхности дель Пеццо .................. 74

3.2. Минимальные поверхности дель Пеццо ................... 77

4. Бирациональная жесткость: основная гипотеза ................ 78

4.1. Расслоения с нерациональной базой ..................... 78

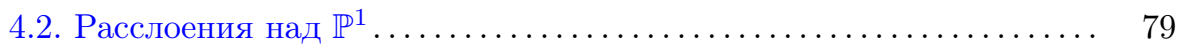

4.3. Перестройки слоя и "хорошие" модели ................... 82

5. Расслоения на поверхности дель Пеццо степени 1 . ............... 89

5.1. Конструкция через двойное накрытие .................. 89

5.2 . Конструкция через вложение в $\mathbb{P}_{\mathbb{P}^{1}}(1,1,2,3) \ldots \ldots \ldots \ldots \ldots \ldots . \ldots 9$

5.3. Бирационально жесткие расслоения .................. 95

5.4. Нежесткие случаи ............................. 96

6. Расслоения на поверхности дель Пеццо степени $2 \ldots \ldots \ldots \ldots \ldots \ldots \ldots . . \ldots 9$

6.1. Конструкция через двойное накрытие ..................... 99

6.2. Вложение в $\mathbb{P}(1,1,1,2)$-расслоение .................... 102

6.3. Результаты о жесткости............................ 103

7. Расслоения на кубические поверхности . . . . . . . . . . . . . . . . 106

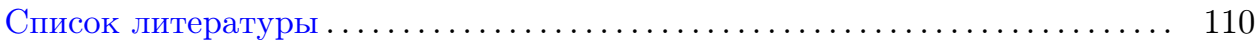

Работа выполнена при поддержке грантов РФФИ 05-01-00353-а, НШ-9969.2006.1, МД4261.2006.1 и CRDF \#RUM1-2692-MO-05.

(C) М. М. Гриненко, 2006 


\section{Введение}

Бирациональная геометрия трехмерных расслоений на поверхности дель Пеццо является важной частью общей бирациональной теории алгебраических многообразий отрицательной кодаировой размерности. И хотя такие расслоения являются естественным обобщением поверхностей дель Пеццо, т.е. двумерной ситуации, на случай, когда в качестве основного поля мы берем поле функций кривой, долгое время все результаты так или иначе сводились именно к такому пониманию и, по сути дела, исчерпывались результатами Ю. И. Манина и В. А. Исковских о поверхностях над незамкнутыми совершенными полями [1]-[3]. Позже иногда удавалось решать проблему рациональности некоторых расслоений на поверхности дель Пеццо методом вырождений [4] или, например, таким экстравагантным способом, как спуск в характеристику и взятие чисто несепарабельных накрытий [5]. Однако систематически теория начала развиваться, как только А. В. Пухликову удалось приспособить метод максимальных особенностей к исследованию расслоений на поверхности дель Пеццо [6].

В данном обзоре мы придерживаемся точки зрения, естественно возникающей из программы Саркисова. Согласно ей, мы исследуем все множество расслоений Мори, бирационально эквивалентных данному многообразию, факторизованное по отношению "бирациональности над базой", т.е. для многообразия $X$ рассматриваем его множество структур Мори $\mathscr{M} \mathscr{S}(X)$. Такая точка зрения имеет как теоретический фундамент в виде программы минимальных моделей и программы Саркисова, так и практический в лице метода максимальных особенностей.

Всюду в данной работе, если специально не оговорено иное, предполагается, что мы рассматриваем многообразия над алгебраически замкнутым полем характеристики 0. Необходимые определения и понятия из программы минимальных моделей, которыми мы будем пользоваться, можно найти в [7].

\section{1. Структуры Мори}

Предположим, что мы имеем неособое трехмерное многообразие $X$ и бирациональное отображение $X \rightarrow V$, являющееся результатом применения к $X$ программы минимальных моделей. В любом случае $V$ - проективное многообразие с $\mathbb{Q}$-факториальными терминальными особенностями, т.е. принадлежащее категории Мори. Но, в зависимости от кодаировой размерности $\kappa(X)$, $V$ будет либо так называемой минимальной моделью (канонический дивизор численно эффективен), либо расслоением Мори.

ОПРЕДЕЛЕНиЕ 1.1. Тройка $\mu: V \rightarrow S$ называется расслоением Мори, если $V$ принадлежит категории Мори, $S$ - нормальное многообразие размерности строго меньшей, чем размерность $V$, и $\mu$ - экстремальное стягивание расслоенного типа, т.е. относительное число Пикара $\rho(V / S)=\rho(V)-\rho(S)$ равно 1 и $\left(-K_{V}\right)$ относительно обилен.

В дальнейшем, чтобы не загромождать текст, расслоения Мори могут обозначаться более компактно. Например, $V / S$ или даже просто $V$ для расслоения Мори $\mu: V \rightarrow S$, если из контекста ясно, о каких структурных морфизмах или базах идет речь. 
В размерности 3 (на данный момент наивысшая размерность, где доказана программа минимальных моделей) есть три возможных типа расслоений Мори:

(a) многообразие Фано, если $\operatorname{dim} S=0$, т.е. $S$ - точка;

(b) расслоение на поверхности дель Пеццо степени $d$, когда $\operatorname{dim} S=1$ (в этом случае слой над общей (схемной) точкой $S$ есть минимальная поверхность дель Пеццо степени $d)$;

(c) расслоение на коники, если $S$ поверхность (слой над общей точкой $S$ будет коникой).

Между расслоениями Мори можно ввести отношение эквивалентности, а через него следующее важное понятие.

ОПРЕДЕЛЕНИЕ 1.2. Мы говорим, что бирациональное отображение $\chi: V \rightarrow$ $V^{\prime}$ между расслоениями Мори $\rho: V \rightarrow S$ и $\rho^{\prime}: V^{\prime} \rightarrow S^{\prime}$ бирационально над базой, а сами расслоения имеют одну и ту же структуру Мори (посредством отображения $\chi$ ), если:

1) $\chi$ проводится через базу, т.е. существует бирациональное отображение $\psi: S \rightarrow S^{\prime}$ такое, что $\rho^{\prime} \circ \chi=\psi \circ \rho$;

2) индуцированное отображение $\chi_{\eta}: V_{\eta} \rightarrow V_{\eta}^{\prime}$ слоев над общей схемной точкой $\eta$ многообразия $S$ (или $S^{\prime}$, что в данном случае одно и то же) является, на самом деле, бирациональным автоморфизмом (другими словами, $V_{\eta}$ и $V_{\eta}^{\prime}$ бирегулярно изоморфны и $\left.\chi_{\eta} \in \operatorname{Bir}\left(V_{\eta}\right)\right)$.

Указанное отношение (т.е. "быть бирациональным над базой") разбивает все множество расслоений Мори, бирационально эквивалентных некоторому многообразию $X$, на классы эквивалентности, и множество этих классов эквивалентности мы будем обозначать $\mathscr{M} \mathscr{S}(X)$ и называть множеством структур Мори на $X$.

Смысл этого определения заключается в том, чтобы рассматривать не вообще все расслоения Мори, бирациональные некоторому многообразию, а только существенно различные. Например, если расслоение на коники $V^{\prime} / S^{\prime}$ получено из расслоения на коники $V / S$ путем раздутия слоя (в общем случае это неприводимая коника) над некоторой точкой базы (при этом $S^{\prime}$ есть раздутие $S$ в соответствующей точке), то с точки зрения определения 1.2 расслоения $V / S$ и $V^{\prime} / S^{\prime}$ отличаются несущественно и представляют одну и ту же структуру Мори.

Определение 1.2 требует некоторых пояснений. Пусть $V$ и $V^{\prime}$ - многообразия Фано, т.е. $S$ и $S^{\prime}$ - точки. Тогда отображение $\psi$ тривиально, оно просто отождествляет эти точки. При этом $\eta$ отождествляется с $S$ и $S^{\prime}$, а слои $V_{\eta}$ и $V_{\eta}^{\prime}$ - это сами многообразия Фано $V$ и $V^{\prime}$. Таким образом, определение требует, чтобы $V$ и $V^{\prime}$ были изоморфны, так что $\chi \in \operatorname{Bir}(V)$. С этой точки зрения $\mathbb{P}^{3}$ и трехмерная квадрика, будучи бирационально изоморфными, представляют различные структуры Мори на $\mathbb{P}^{3}$.

Предположим, что $S$ и $S^{\prime}$ - кривые (неособые), так что $V / S$ и $V^{\prime} / S^{\prime}$ - pacслоения на поверхности дель Пеццо. Тогда если $\chi$ бирационально над базой, то $\psi$ - изоморфизм кривых, а поверхности $V_{\eta}$ и $V_{\eta}^{\prime}$ изоморфны. Тонкость ситуации заключается в том, что если $\chi$ проводится через базу (но не оговорен пункт 2) определения), может сложиться ситуация, когда $\chi_{\eta}: V_{\eta}-\rightarrow V_{\eta}^{\prime}$ задает отображение между неизоморфными (бирегулярно) поверхностями дель Пеццо, причем последние могут иметь как одинаковые, так и разные степени (об 
этом речь пойдет в разделе 3), и в таком случае естественно полагать, что мы имеем различные структуры Мори.

Проще всего с расслоениями на коники, так как в этом случае индуцированное отображение $\chi_{\eta}$, очевидно, всегда будет изоморфизмом, так что второй пункт определения в этой ситуации является лишним.

Множество структур Мори на протяжении всей статьи рассматривается как основной бирациональный инвариант многообразий отрицательной кодаировой размерности. В дальнейшем условимся обозначать класс расслоения Мори $\rho: V \rightarrow S$ в множестве $\mathscr{M} \mathscr{S}(V)$ через $[\rho: V \rightarrow S]$.

Имеется один важный частный случай, когда множество структур Мори выглядит особенно просто.

ОПРЕДЕЛЕНИЕ 1.3. Будем говорить, что многообразие $X$ бирационально жестко (или просто жестко), если $\mathscr{M} \mathscr{S}(X)$ содержит ровно один элемент. Например, расслоение Мори $\rho: V \rightarrow S$ бирационально жестко, если

$$
\mathscr{M} \mathscr{S}(V)=\{[\rho: V \rightarrow S]\} .
$$

Расслоение Мори $V / S$ называется сверхжестким, если любое его бирациональное отображение на расслоение Мори является изоморфизмом; в частности, для таких расслоений Мори $\operatorname{Bir}(V)=\operatorname{Aut}(V)$.

Жесткие многообразия составляют класс многообразий отрицательной кодаировой размерности, по своим бирациональным свойствам наиболее далекий от $\mathbb{P}^{3}$. В частности, все такие многообразия нерациональны по очевидным причинам $\left(\mathscr{M} \mathscr{S}\left(\mathbb{P}^{3}\right)\right.$ содержит очень много элементов). Сверхжесткость же является предельным случаем бирациональной жесткости: любое бирациональное отображение сверхжесткого расслоения Мори является изоморфизмом.

Отметим, что существует и другое определение бирациональной жесткости [8], оперирующее понятием порога канонического присоединения и по смыслу, вообще говоря, отличающееся от данного выше. Однако понимание жесткости как единственности структуры Мори геометрически более прозрачно, и именно такое определение мы будем использовать в этой работе.

В статье далее будут, главным образом, рассматриваться следующие вопросы бирациональной геометрии расслоений на поверхности дель Пеццо: проблема рациональности, проблема бирациональной жесткости, описание множества структур Мори. Завершим этот раздел некоторыми примерами, иллюстрирующими введенные выше понятия и касающимися, в основном, многообразий Фано и расслоений на коники.

ПримеР 1.4 (квартики в $\left.\mathbb{P}^{4}\right)$. Во многих отношениях базовым примером является неособая гиперповерхность $X_{4} \subset \mathbb{P}^{4}$ степени 4. В основополагающей работе [9] В. А. Исковских и Ю. И. Маниным было доказано, что такие квартики сверхжестки.

Что будет, если мы начнем вырождать неособую квартику? Рассмотрим несколько примеров. Пусть $X_{4}$ будет квартикой, имеющей ровно одну обыкновенную двойную точку (в подходящих локальных координатах такая особая точка задается уравнением $x y+z w=0)$. Предположим, что $X_{4}$ является общей в следующем смысле: через особую точку проходит ровно 24 прямых. В этих предположениях А. В. Пухликов доказал [10], что $X_{4}$ бирационально жестко, но 
уже не сверхжестко. Существуют 24 бирациональные инволюции, связанные с прямыми, проходящими через особую точку, и еще одна инволюция, устроенная так: общая прямая, проходящая через особую точку, пересекает $X_{4}$ еще в двух точках, и эта инволюция просто переставляет их. Показано, что между этими инволюциями нет соотношений.

Будем вырождать $X_{4}$ дальше и потребуем, чтобы она содержала единственную особую точку вида $x y+z^{2}+w^{n}=0$ при $n \geqslant 3$. Для таких квартик существует гипотеза [11], что они являются жесткими. Но если мы "испортим" особую точку иным образом, потребовав ее локального уравнения $x y+z^{3}+w^{3}=0$, то в предположениях общности такой квартики А. Корти и М. Мелла [11] доказали, что $X_{4}$ бирационально изоморфна некоторому многообразию Фано $Y_{3,4} \subset \mathbb{P}\left(1^{4}, 2^{2}\right)$, являющемуся полным пересечением кубики и квартики. Более того, в этом случае $\mathscr{M} \mathscr{S}\left(X_{4}\right)=\left\{\left[X_{4}\right],\left[Y_{3,4}\right]\right\}$. Как видно, это уже нежесткое многообразие.

Существует еще один путь вырождения квартики, приводящий к нежестким многообразиям. Именно, потребуем, чтобы квартика содержала поверхность, не высекаемую никаким дивизором в $\mathbb{P}^{4}$, но при этом сохраняла терминальность особенностей. Простейшим примером такой поверхности является плоскость $P$. Итак, пусть мы имеем $P \subset X_{4} \subset \mathbb{P}^{4}$. Очевидно, $X_{4}$ не лежит в категории Мори, но общая такая квартика содержит 9 обыкновенных двойных точек. Все они лежат на $P$. Можно показать, что в общем случае $\mathscr{M} \mathscr{S}\left(X_{4}\right) \supset\left\{\left[Y_{3,3}\right],\left[V / \mathbb{P}^{1}\right]\right\}$, где $Y_{3,3} \subset \mathbb{P}\left(1^{5}, 2\right)$ - полное пересечение двух кубик, а $V / \mathbb{P}^{1}$ - неособое расслоение на кубические поверхности. Другим примером является случай $Q \subset X_{4} \subset \mathbb{P}^{4}$, где $Q$ - квадратичная поверхность. Такая квартика также не лежит в категории Мори и терминальна, содержит 12 двойных точек. В общем случае $\mathscr{M} \mathscr{S}\left(X_{4}\right) \supset\{[A],[B]\}$, где $A$ и $B$ - многообразия Фано, изоморфные полному пересечению квадрики и кубики в $\mathbb{P}^{5} \mathrm{c}$ одной двойной точкой. Подобные примеры с некоторыми результатами о нерациональности можно найти в обзоре И. А. Чельцова [12]. Отметим, что если квартика с плоскостью содержит еще $c D 4$-точку вне плоскости, то она бирационально изоморфна многообразию Фано индекса 2 и степени 2 , имеющему еще большее разнообразие структур Мори (теорема 6.4, расслоения на поверхности дель Пеццо степени 2 с набором $(0,0,1))$. Такие квартики содержат уже континуум различных структур Мори.

ПРимеР 1.5 (расслоения на коники). Известно [13], [14], что любое расслоение на коники может быть бирационально над базой преобразовано в стандартное расслоение на коники $V / S$, т.е. к ситуации, когда $V / S$ - расслоение Мори и $V$ и $S$ неособы. В таких условиях В. Г. Саркисов показал [15], что если линейная система $\left|4 K_{S}+C\right|$, где $C$ - дискриминантная кривая, непуста, то $V$ бирационально жестко. Существует, однако, гипотеза, что $V / S$ жестко тогда и только тогда, когда $\left|3 K_{S}+C\right| \neq \varnothing$. Кроме того, В. А. Исковских был сформулирован гипотетический критерий рациональности для стандартных расслоений на коники [16], [17]. К сожалению, эти гипотезы, особенно последняя, пока очень далеки от доказательства. 


\section{2. Программа Саркисова и метод максимальных особенностей}

Программа Саркисова - это общетеоретический фундамент в исследованиях расслоений Мори и бирациональных отображений между ними. Она сформулирована и доказана в размерности 3 [18]. В статье будут использованы два основных момента этой теории. Прежде всего, это теорема, имеющая важное теоретическое и прикладное значение.

ТЕорема 2.1 [18; теорема 4.2]. Пусть $\chi: V \rightarrow V^{\prime}-$ бирациональное отображение расслоений Мори $\rho: V \rightarrow S$ и $\rho^{\prime}: V^{\prime} \rightarrow S^{\prime}$. Пусть $\mathscr{D}^{\prime}=\mid n^{\prime}\left(-K_{V^{\prime}}\right)+$ $\rho^{* *}\left(A^{\prime}\right) \mid$ - оченъ обильная линейная система, где $A^{\prime}$ - обильный дивизор на $S^{\prime}$, u $\mathscr{D}=\chi_{*}^{-1} \mathscr{D}^{\prime} \subset\left|n\left(-K_{V}\right)+\rho^{*}(A)\right|-$ ее собственный прообраз, $A-$ некоторый дивизор на $S$. Тогда:

1) $n \geqslant n^{\prime}$, и если $n=n^{\prime}$, то ұ бирачионально над базой (m.е. $V / S$ и $V^{\prime} / S^{\prime}$ представляют одну и ту же структуру Мори);

2) если пара $K_{V}+\frac{1}{n} \mathscr{D}$ канонична и численно эфбективна (т.е. дивизор $A$ численно эфффективен), то ұ - изоморфизм.

Другой момент связан с факторизацией бирациональных отображений. Пусть даны два трехмерных расслоения Мори $V / S$ и $V^{\prime} / S^{\prime}$ и бирациональное отображение $\chi: V \rightarrow V^{\prime}$. Тогда программа Саркисова утверждает [18], что существует конечная цепочка бирациональных отображений

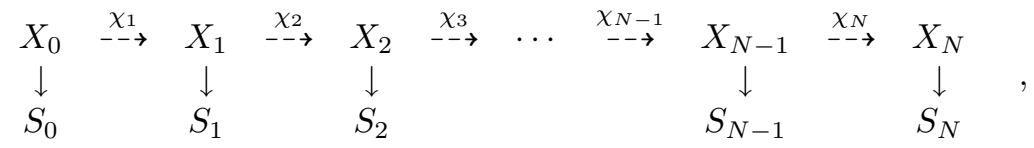

где $V / S=X_{0} / S_{0}, X_{1} / S_{1}, \ldots, X_{N} / S_{N}=V^{\prime} / S^{\prime}$ - расслоения Мори, такая, что $\chi=\chi_{N} \circ \chi_{N-1} \circ \cdots \circ \chi_{2} \circ \chi_{1}$ и каждый $\chi_{i}$ принадлежит одному из четырех элементарных линков (Саркисова). Эти линки изображены на рис. 1 (для примера показаны все возможные разложения отображения $\chi_{2}: X_{1} \rightarrow X_{2}$ ).

На этом рисунке во всех типах линков $\psi$ - изоморфизм в коразмерности 1 (последовательность лог-флипов). Для линка типа I $\mu$ обозначает морфизм со связными слоями и $\gamma$ - экстремальное дивизориальное стягивание. Отметим, что $\rho\left(S_{2} / S_{1}\right)=1$. Для типа II $\gamma_{1}$ и $\gamma_{2}$ - экстремальные дивизориальные стягивания и $\mu$ - бирациональное отображение. Линк типа III - это обращение типа I. Наконец, в линках типа IV $\delta_{1}$ и $\delta_{2}-$ морфизмы со связными слоями, $R-$ нормальное многообразие и $\rho\left(S_{1} / R\right)=\rho\left(S_{2} / R\right)=1$. Отметим, что разложение отображения на линки неоднозначно. В частности, композиция элементарных линков может быть элементарным линком.

Сам процесс факторизации устроен следующим образом. Для расслоения Мори $\rho: V \rightarrow S$ и линейной системы без неподвижных компонент $\mathscr{D} \subset$ $\left|n\left(-K_{V}\right)+\rho^{*}(A)\right|$, где $A-$ некоторый дивизор на $S$, определяется тройка неотрицательных чисел $(n, c, e)$. В ней $c$ - канонический порог, т.е. такое максимальное рациональное число, что пара $K_{V}+c \mathscr{D}$ канонична, а $e$ - число $\left(K_{V}+c \mathscr{D}\right)$-крепантных нормирований (равное относительному числу Пикара максимального крепантного разрешения для пары $\left.K_{V}+c \mathscr{D}\right)$. На указанных тройках вводится частичный порядок: $(n, c, e)>\left(n_{1}, c_{1}, e_{1}\right)$, если $n>n_{1}$, либо $n=n_{1}$ и $c_{1}>c$, либо $n=n_{1}, c=c_{1}$ и $e>e_{1}$. 


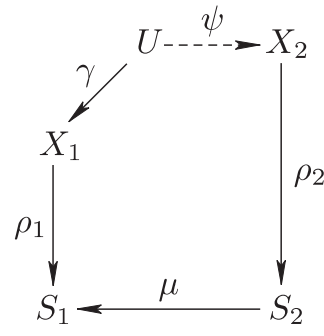

I

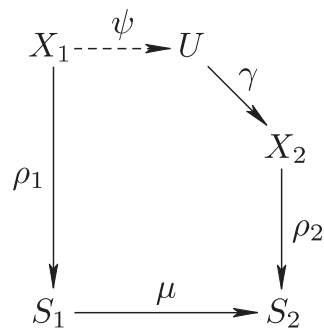

III

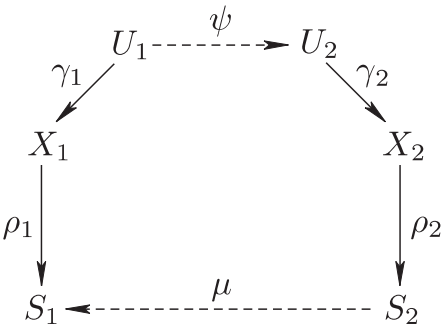

II

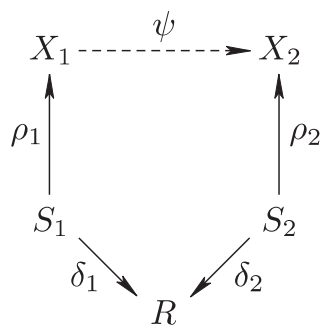

IV

Рис. 1. Элементарные линки Саркисова

Предположим, что $\mathscr{D}$ является собственным прообразом линейной системы $\mathscr{D}^{\prime}$ на $V^{\prime} / S^{\prime}$, удовлетворяющей условиям теоремы 2.1 , и пусть пара $K_{V}+c \mathscr{D}$ неканонична, т.е. $c<\frac{1}{n}$. Это означает, что существует дискретное нормирование $\mathfrak{v}$, реализуемое экстремальным раздутием $\gamma: U \rightarrow V$, такое, что $\mathfrak{v}(\mathscr{D})>$ $n \delta_{\mathfrak{v}}$ (неравенство Нётера-Фано). В этом неравенстве $\delta_{\mathfrak{v}}-$ каноническая кратность (дискрепанта) относительно нормирования $\mathfrak{v}$, другими словами, $K_{U}=$ $\gamma^{*}\left(K_{V}\right)+\delta_{\mathfrak{v}} E$ для исключительного дивизора $E$ экстремального раздутия $\gamma$. В таком случае показывается, что существует линк $\chi_{1}: V / S=X_{0} / S_{0}-\rightarrow X_{1} / S_{1}$ типа I или II, при этом $(n, c, e)>\left(n_{1}, c_{1}, e_{1}\right)$. Если же пара $K_{V}+c \mathscr{D}$ канонична, т.е. $c \geqslant \frac{1}{n}$, то мы полагаем, что дивизор $A$ не численно эффективен на $S$ (в противном случае нечего факторизовать, что следует из теоремы 2.1). Тогда существует линк $\chi_{1}: V / S=X_{0} / S_{0} \rightarrow X_{1} / S_{1}$ типа III или IV. Далее мы рассматриваем собственный образ $\mathscr{D}$ на $X_{1}$ и продолжаем процедуру. В [18] показано, что последовательность линков обрывается на изоморфизме.

Программа Саркисова так и осталась бы общей теорией, если бы не оказалось, что появившийся задолго до нее метод максимальных особенностей [8] предоставляет необходимый набор приемов для ее практической реализации. Связь между ними заключается в следующем. Максимальным нормированием для линейной системы $\mathscr{D}$ на многообразии $X$ в методе максимальных особенностей называется геометрическое (т.е. реализуемое одним из исключительных дивизоров некоторого бирационального морфизма) дискретное нормирование $\mathfrak{v}$ такое, что выполнено неравенство Нётера-Фано $\mathfrak{v}(\mathscr{D})>n \delta_{\mathfrak{v}}\left(\delta_{\mathfrak{v}}\right.$, как и выше, - каноническая кратность). Максимальной особенностью линейной 
системы $\mathscr{D}$ называется пара $(B, \mathfrak{v})$, где $\mathfrak{v}$ - максимальное нормирование для $\mathscr{D}$ и $B=$ Center $_{X} \mathfrak{v}$. Предполагая $X$ принадлежащим категории Мори, можно показать, что если $\mathscr{D}$ имеет максимальную особенность, то для $\mathscr{D}$ также существует и максимальная особенность $\left(B^{\prime}, \mathfrak{v}^{\prime}\right)$ такая, что $B^{\prime} \subset B$ и $\mathfrak{v}^{\prime}$ реализуется экстремальным раздутием. Другими словами, мы в исходной ситуации для линков типа I или II. Тогда, следуя разработанным приемам метода максимальных особенностей, мы либо доказываем нереализуемость такой ситуации, либо предъявляем конкретный линк типа I или II, как в программе Саркисова, тем самым "откручивая" (в терминологии метода) максимальную особенность. Остается только добавить, что замены $(B, \mathfrak{v})$ на $\left(B^{\prime}, \mathfrak{v}^{\prime}\right)$ может и не найтись, если мы имеем дело со сверхмаксимальными особенностями, которые нужны для работы с расслоениями на поверхности дель Пеццо и для которых выполняется усиленное неравенство Нётера-Фано.

\section{3. Поверхности дель Пеццо}

В этом разделе собраны необходимые сведения о поверхностях дель Пеццо. Под поверхностью дель Пеццо понимается (неприводимая) алгебраическая поверхность $F$ с антиобильным дуализирующим пучком. Другими словами, на поверхности определен канонический дивизор Вейля $K_{F}$ такой, что $-K_{F}$ обилен. Во всех ситуациях, которые встретятся, $K_{F}$ является $\mathbb{Q}$-Картье дивизором (т.е. некоторая его целая кратность есть дивизор Картье). Под степенью поверхности дель Пеццо понимается число $d=K_{F}^{2}$. Кроме того, почти всегда мы будем иметь дело с горенштейновыми случаями, т.е. уже $K_{F}$ является дивизором Картье. При этом степень $d$ будет, очевидно, целым числом, причем $1 \leqslant d \leqslant 9$.

Поверхности дель Пеццо в расслоении Мори $\rho: V \rightarrow S$ над кривой $S$ бывают двух "видов": как слой над некоторой точкой $S$ и как слой над общей схемной точкой $S$. Соответственно, материал разбит на две группы: горенштейновы поверхности дель Пеццо над основным (алгебраически замкнутым) полем и неособые минимальные поверхности дель Пеццо над незамкнутым полем (полем функций кривой $S$ ).

3.1. Горенштейновы поверхности дель Пеццо. Пусть $F$ - неприводимая горенштейнова поверхность дель Пеццо. Известно, что тогда либо $F$ неособа, либо $F$ имеет только дювалевские особенности, либо единственную минимальную эллиптическую особую точку, либо $F$ ненормальна. Для нас важно, что во всех этих случаях отображения, определяемые антиканоническими линейными системами и их кратностями, будут устроены одинаково [19]-[22].

ПРЕДЛОЖЕНИЕ 3.1. Пусть $F$ - горенштейнова неприводимая поверхность дель Пецио степени $d$. Если $3 \leqslant d \leqslant 9$, то линейная система $\left|-K_{F}\right|$ очень обильна и определяет вложение $\varphi: F \rightarrow \mathbb{P}^{d}$. При $d=9$ поверхность $F$ есть $\mathbb{P}^{2}$ и $\varphi$ - вложение при помощи полной системъ плоских кубик. При $d=8$ $F$ изоморфна либо двумерной квадрике в $\mathbb{P}^{3}$, либо поверхности $\mathbb{F}_{1}$. При $d=3$ поверхность $F \subset \mathbb{P}^{3}$ задается однородным неприводимым многочленом степени 3. При $d=4$ поверхность $F$ является полным пересечением двух квадрик в $\mathbb{P}^{4}$. 
Пусть $d=1$. Тогда линейная система $\left|-K_{F}\right|$ имеет единственную базисную точку, в которой $F$ неособа, система $\left|2\left(-K_{F}\right)\right|$ свободна от базисных точек, а $\left|3\left(-K_{F}\right)\right|$ очень обильна и вкладывает $F$ в $\mathbb{P}^{6}$ как поверхность степени 9. При подходящем выборе координат $[x, y, z, w]$ весов $(1,1,2,3)$ в $\mathbb{P}(1,1,2,3)$ поверхность $F$ задается уравнением шестой степени

$$
w^{2}+z^{3}+z f_{4}(x, y)+f_{6}(x, y)=0
$$

$f_{i}$ - однородные полиномы степени $i$. Линейная система $\left|2\left(-K_{F}\right)\right|$ задает морфизм

$$
\varphi: F \stackrel{2: 1}{\longrightarrow} R_{Q} Q \subset \mathbb{P}^{3}
$$

степени 2 , где $Q$ - невырожденный квадратичный конус в $\mathbb{P}^{3}, R_{Q}=\left.R\right|_{Q}-\partial u$ визор ветвления, а $R \subset \mathbb{P}^{3}$ - кубическая поверхность, не содержащая вершину конуса $Q$.

Пусть $d=2$. Тогда $\left|-K_{F}\right|$ не имеет базисньх точек, $\left|2\left(-K_{F}\right)\right|$ очень обильна и вкладывает $F$ в $\mathbb{P}^{6}$ как поверхность степени 8. При подходящем выборе координат $[x, y, z, w]$ с весами $(1,1,1,2)$ в $\mathbb{P}(1,1,1,2)$ поверхность $F$ задается уравнением четвертой степени

$$
w^{2}+f_{4}(x, y, z)=0
$$

где $f_{4}$ - однородный полином степени 4. Линейная система $\left|-K_{F}\right|$ определяет морфизм

$$
\varphi: F \stackrel{2: 1}{\longrightarrow}_{R} \mathbb{P}^{2}
$$

степени 2, разветвленный над кривой $R \subset \mathbb{P}^{2}$ степени 4.

Разберем подробнее конструкции поверхностей дель Пеццо степеней 1 и 2. Рассмотрим лишь неособые случаи, ситуация особых поверхностей разбирается в [21] и [22] (хотя принципиально устроена так же).

Начнем с $d=1$. Пусть $F$ - неособая поверхность дель Пеццо степени $d=\left(-K_{F}\right)^{2}=1$. Нетрудно видеть, что все элементы линейной системы $\left|-K_{F}\right|$ приведены и неприводимы, общий неособ и это кривые арифметического рода 1 и степени 1 (относительно пересечения с $-K_{F}$ ). Кроме того, $\left|-K_{F}\right|$ имеет единственную базисную точку $P$. Выберем неособую кривую $C \in\left|-K_{F}\right|$. Точная последовательность

$$
0 \longrightarrow \mathscr{O}_{F}\left(-(i-1) K_{F}\right) \longrightarrow \mathscr{O}_{F}\left(-i K_{F}\right) \longrightarrow \mathscr{O}_{C}\left(-i K_{F}\right) \longrightarrow 0
$$

и теорема Кодаиры об обращении в нуль дают для всех $i \geqslant 0$ эпиморфизмы

$$
H^{0}\left(F,-i K_{F}\right) \longrightarrow H^{0}\left(C,\left.\left(-i K_{F}\right)\right|_{C}\right) \longrightarrow 0 .
$$

Получаем сюръективное отображение градуированных алгебр

$$
\mathscr{A}_{F}=\bigoplus_{i \geqslant 0} H^{0}\left(F,-i K_{F}\right) \longrightarrow \mathscr{A}_{C}=\bigoplus_{i \geqslant 0} H^{0}\left(C,\left.\left(-i K_{X}\right)\right|_{C}\right),
$$

сохраняющее градуировку. Алгебра $\mathscr{A}_{C}$, а значит, и алгебра $\mathscr{A}_{F}$ порождены элементами степени не выше 3. Учитывая, что

$$
h^{0}\left(X,-K_{F}\right)=2, \quad h^{0}\left(X,-2 K_{F}\right)=4, \quad h^{0}\left(X,-3 K_{F}\right)=7,
$$


можно определить следующие порождающие:

$$
\begin{gathered}
H^{0}\left(F,-K_{F}\right)=\mathbb{C}\langle x, y\rangle, \\
H^{0}\left(F,-2 K_{F}\right)=\mathbb{C}\left\langle x^{2}, x y, y^{2}, z\right\rangle, \\
H^{0}\left(F,-3 K_{F}\right)=\mathbb{C}\left\langle x^{3}, x^{2} y, x y^{2}, y^{3}, z x, z y, w\right\rangle,
\end{gathered}
$$

где $x$ и $y$ имеют вес 1 , а $z$ и $w$ - веса 2 и 3 соответственно. Таким образом, $F$ вкладывается во взвешенное проективное пространство:

$$
F=\operatorname{Proj} \mathscr{A}_{F}=\operatorname{Proj} \mathbb{C}[x, y, z, w] / I_{F} \subset \mathbb{P}(1,1,2,3),
$$

где $I_{F}$ - главный идеал, порожденный элементами степени 6. Действительно, $h^{0}\left(F,-6 K_{F}\right)=22$, но однородные элементы степени 6 в $\mathbb{C}[x, y, z, w]$ образуют пространство размерности 23 , так что имеем одно линейное соотношение, которое и порождает идеал $I_{F}$. Так как $F$ не проходит через особые точки $\mathbb{P}(1,1,2,3)$, уравнение поверхности всегда может быть записано в виде

$$
F=\left\{w^{2}+z^{3}+z f_{4}(x, y)+f_{6}(x, y)=0\right\} \subset \mathbb{P}(1,1,2,3),
$$

где $f_{i}$ - однородные полиномы степени $i$. Кроме того, рассмотрим проекцию

$$
\mathbb{P}(1,1,2,3) \rightarrow \mathbb{P}(1,1,2)
$$

из точки $0: 0: 0: 1$, не лежащей на $F$. Ограничение этой проекции на $F$ дает морфизм степени 2

$$
\varphi: F \rightarrow \mathbb{P}(1,1,2) .
$$

Поверхность $\mathbb{P}(1,1,2)$ изоморфна невырожденному квадратичному конусу $Q \subset \mathbb{P}^{3}$. Точка $P=\operatorname{Bas}\left|-K_{F}\right|$ лежит в точности над вершиной $Q$. Морфизм $\varphi$ разветвлен над неособым сечением $Q$ кубикой и задается линейной системой $\left|2\left(-K_{F}\right)\right|$.

Пусть теперь $F$ - неособая поверхность дель Пеццо степени 2. Рассуждения близки к предыдущему случаю. Нетрудно показать, что линейная система $\left|-K_{F}\right|$ не имеет базисных точек и содержит неособый элемент $C$. Как и выше, имеем сюръективный морфизм градуированных алгебр $\mathscr{A}_{F} \rightarrow \mathscr{A}_{C}$, но теперь эти алгебры порождены элементами степени не выше 2. Далее, $h^{0}\left(F,-K_{F}\right)=3$ и $h^{0}\left(F,-2 K_{F}\right)=7$, и мы полагаем

$$
\begin{gathered}
H^{0}\left(F,-K_{F}\right)=\mathbb{C}\langle x, y, z\rangle, \\
H^{0}\left(F,-2 K_{F}\right)=\mathbb{C}\left\langle x^{2}, y^{2}, z^{2}, x y, x z, y z, w\right\rangle,
\end{gathered}
$$

где $x, y$ и $z$ имеют вес 1 , а $w$ - веса 2 . Тогда

$$
F=\operatorname{Proj} \mathscr{A}_{F}=\operatorname{Proj} \mathbb{C}[x, y, z, w] / I_{X} \subset \mathbb{P}(1,1,1,2),
$$

где главный идеал $I_{F}$ порожден однородным элементом степени 4, поскольку $h^{0}\left(F,-4 K_{F}\right)=21$, в то время как размерность пространства однородных элементов $\mathbb{C}[x, y, z, w]$ степени 4 равна 22 . Так как $F$ не проходит через особую точку $\mathbb{P}(1,1,1,2)$, поверхность $F$ может быть задана как

$$
F=\left\{w^{2}+f_{4}(x, y, z)=0\right\} \subset \mathbb{P}(1,1,1,2),
$$

где $f_{4}$ - однородный полином степени 4. Проекция $\mathbb{P}(1,1,1,2)-\rightarrow \mathbb{P}(1,1,1)=\mathbb{P}^{2}$ из точки $(0: 0: 0: 1)$, ограниченная на $F$, представляет $F$ в виде двойного накрытия $\mathbb{P}^{2}$, разветвленного над неособой квартикой. Этот морфизм задается линейной системой $\left|-K_{F}\right|$. 
3.2. Минимальные поверхности дель Пеццо. Пусть $k$ - поле характеристики $0, \bar{k}$ - алгебраическое замыкание, $G=\operatorname{Gal}(\bar{k} / k)$ - группа Галуа. Под полем $k$ можно подразумевать, например, поле функций некоторой кривой, это как раз наш случай. Пусть $F$ - минимальная поверхность дель Пеццо степени $d=K_{F}^{2}, \operatorname{Pic}(F) \cong \mathbb{Z}$, и $F_{\bar{k}}=F \otimes \bar{k}-$ соответствующая поверхность дель Пеццо над $\bar{k}$.

Заметим, что $d \neq 7$. Действительно, $F_{\bar{k}}$ изоморфна раздутию $\mathbb{P}_{\bar{k}}^{2}$ в двух точках, так что содержит три исключительные кривые $e_{0}, e_{1}$ и $e_{2}$. Мы полагаем, что $e_{0}$ пересекает кривые $e_{1}$ и $e_{2}$, не имеющие общих точек. Очевидно, если раздуваемые на плоскости точки неподвижны при действии $G$, т.е. определены над $k$, то $F$ не минимальна. Значит, $G$ переставляет эти точки на плоскости, а значит, переставляет $e_{1}$ и $e_{2}$. Но тогда $e_{0}$ неподвижна при действии $G$, стало быть, определена над $k$. Поэтому $F$ не минимальна.

Заметим также, что $F$ всегда имеет рациональные точки, т.е. точки, определенные над $k$ [23]. Более того, в ситуации, когда $k$ является полем функций кривой, показано, что $F$, на самом деле, имеет всюду плотное по Зарисскому множество рациональных точек [24; теорема 2.13]. С точки зрения трехмерного расслоения на поверхности дель Пеццо $V / S$ это означает, что существует достаточно много сечений.

Первая часть нужной нам информации о минимальных поверхностях дель Пеццо объединена в следующем предложении.

ПреДЛОЖЕНИЕ 3.2. Пусть $F$ - минималъная поверхность дель Пецио над $k$. Тогда:

1) если $d \geqslant 5$, то $F$ рациональна над $k[1$; следствие 3 к теореме 3.7 ; следствие 2 к теореме 3.10; теорема 3.15$]$;

2) если $1 \leqslant d \leqslant 3$, то $F$ нерациональна над $k$ [2; теорема 5.7];

3) конструкции поверхностей дель Пеццо из предложения 3.1 годятся и для $F$.

Этот раздел мы завершим важными для последующего изложения результатами. Заметим, что, по аналогии с трехмерным случаем, можно рассматривать программу Саркисова для поверхностей и элементарные линки, определяемые так же, как на рис. 1, только без лог-флипов $\psi$, так что изоморфизм в коразмерности 1 для поверхностей есть изоморфизм. В двумерном случае эта программа также доказана $[25 ; \S 2]$. Нас интересуют отображения между двумя классами поверхностей: минимальными поверхностями дель Пеццо (класс D) и относительно минимальными расслоениями на коники (класс $\mathbf{C}$ ).

ПреДЛОЖЕНИЕ 3.3 [25; теорема 2.6]. Пусть $F$ - минималъная поверхность дель Пецио степени $d, T$ - поверхность класса $\mathbf{D}$ или $\mathbf{C}$ и $\chi: F \rightarrow T-$ элементарный линк. Поверхности и линк считаются определенными над $k$. Тогда: $d=1: T \cong F$ и - изоморфизм;

$d=2: T \cong F u \chi-$ либо изоморфизм, либо инволюиия Бертини с иентром в точке степени 1 ;

$d=3: T \cong F$ и - либо изоморфизм, либо инволючия Бертини в точке степени 2, либо инволюиия Гейзера с иентром в точке степени 1;

$d=4:$ либо $\chi$ - раздутие точки степени 1 на $F$, при этом $T$ - кубическая поверхность с (-1)-кривой над $k$ (поверхность класса $\mathbf{C})$, либо $T \cong F, u$ 
тогда ұ - или изоморфизм, или инволючия Бертини с иентром в точке степени 3, или инволюиия Гейзера с центром в точке степени 2.

Отметим, что при $d \geqslant 5$ возможны нетривиальные линки между неизоморфными минимальными поверхностями дель Пеццо.

Действие инволюций Бертини и Гейзера на дивизорах описано в той же теореме 2.6 из [25]. Инволюция Бертини действует так: мы раздуваем точку степени 1, 2 или 3 соответственно на поверхности дель Пеццо степени 2, 3 или 4, получая при этом поверхность дель Пеццо степени 1. Затем применяем бирегулярную инволюцию двойного накрытия и возвращаемся обратно. Инволюция Гейзера устроена похожим образом: раздуваем точку степени 1 или 2 на поверхности дель Пеццо степени 3 или 4 соответственно, получаем поверхность дель Пеццо степени 2, применяем инволюцию двойного накрытия и возвращаемся обратно.

\section{4. Бирациональная жесткость: основная гипотеза}

4.1. Расслоения с нерациональной базой. Пусть $\rho: V \rightarrow S$ - расслоение на поверхности дель Пеццо степени $d, \eta$ - общая схемная точка (неособой) кривой $S, V_{\eta}$ - слой над общей схемной точкой базы. Тогда, очевидно, $\operatorname{Pic}(V) \otimes \mathbb{Q}=\mathbb{Q}\left[-K_{V}\right] \oplus \rho^{*}(\operatorname{Pic}(S) \otimes \mathbb{Q}) ;$ кроме того, $\left(-K_{V_{\eta}}\right)^{2}=d$ и $\operatorname{Pic}\left(V_{\eta}\right)=$ $\mathbb{Z}\left[-K_{V_{\eta}}\right]$, т.е. $V_{\eta}$ - минимальная неособая поверхность дель Пеццо степени $d$ над полем функций кривой $S$.

Рассмотрим следующую диаграмму:

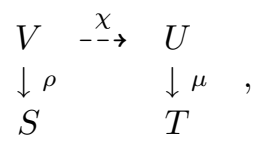

где $\mu: U \rightarrow T$ - некоторое расслоение Мори. Мы хотим узнать об этой ситуации как можно больше. В частности, нас интересует, в каких случаях $\chi$ бирационально над базой, в каких - нет, и т. д.

Предположим для начала, что кривая $S$ нерациональна. Тогда $U$ не может быть многообразием Фано. Аргументировать это можно различным образом, например, используя то, что группа Пикара $V$ содержит непрерывную часть, что невозможно для $U$ или его разрешения особенностей. Или можно воспользоваться тем фактом, что любое многообразие Фано рационально связно [26], в то время как $V / S$ не содержит рациональных кривых, накрывающих базу, т.е. $V$ не рационально связно.

Таким образом, $U / T$ - либо расслоение на поверхности дель Пеццо, либо расслоение на коники. Во всех случаях слои морфизма $\mu$ покрываются рациональными кривыми. Образы этих кривых на $V$ не могут накрывать $S$, тем самым определено рациональное отображение $T \rightarrow S$, на самом деле являющееся морфизмом. Получаем коммутативную диаграмму

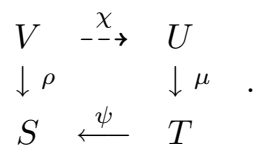


Обозначим $\zeta$ общую схемную точку $T$ и $U_{\zeta}$ слой над ней. Предположим, что $U / T$ - расслоение на поверхности дель Пеццо, т.е. $T$ - кривая. Так как $\chi-$ бирациональный изоморфизм, то $\psi: T \rightarrow S$ - изоморфизм, так что имеем бирациональное отображение $V_{\eta} \rightarrow U_{\zeta}=U_{\eta}$.

Предположим теперь, что $U / T$ - расслоение на коники. Мы, очевидно, можем полагать, что $\chi$ представлено линком Саркисова типа I. Композиция $U \rightarrow T \rightarrow S$ позволяет определить $U_{\eta}$ как слой над общей схемной точкой базы расслоения $U \rightarrow S$. Тогда $U_{\eta}$ - неособая поверхность, расслоенная на коники над рациональной кривой $T_{\eta}$. Так как $\rho(U / T)=\rho\left(U_{\eta} / T_{\eta}\right)=1$, то слои $U_{\eta} \rightarrow T_{\eta}$ не содержат (-1)-кривых.

В любом случае мы приходим к ситуации, когда есть бирациональное отображение $V_{\eta} \rightarrow U_{\eta}$, где $U_{\eta}$ минимальна (или относительно минимальна) и является либо поверхностью дель Пеццо, либо расслоением на коники над рациональной кривой. Ситуация эта полностью изучена в работе [25], теорема 2.6, для всех значений $1 \leqslant d \leqslant 9$ (часть этой теоремы приведена в предложении 3.3 для $1 \leqslant d \leqslant 4)$. В точном соответствии с определением 1.2 мы можем говорить о структурах Мори для указанного класса поверхностей (они исчерпывают двумерные расслоения Мори). Таким образом, из объяснений выше вытекает следующий результат.

ПрЕДЛОЖЕНИЕ 4.1. Пусть $\rho: V \rightarrow S$ - расслоение на поверхности дель Пец, степени d над нерациональной кривой $S$. Тогда элементы множества $\mathscr{M} \mathscr{S}(V)$ находятся во взаимно однозначном соответствии с элементами множества $\mathscr{M} \mathscr{S}\left(V_{\eta}\right)$, где $V_{\eta}$ - слой над общей схемной точкой $\eta$ кривой $S$, и могут быть эфбективно перечислены при помощи процедуры, описанной $в$ работе [25]. В частности, при $1 \leqslant d \leqslant 3$ все эти множества состоят из одного элемента, т.е. $V / S$ бирационально жестко. Напротив, при $d \geqslant 4$ расслоения на поверхности дель Пецио всегда нежестки.

4.2. Расслоения над $\mathbb{P}^{1}$. Всюду далее нас будет интересовать ситуация расслоений $\rho: V \rightarrow \mathbb{P}^{1}$ на поверхности дель Пеццо степени $d$. Сделаем ряд замечаний. Согласно предложению 3.2 , при $d \geqslant 5$ поверхность $V_{\eta}$ над общей схемной точкой $\eta$ прямой рациональна, т.е. бирационально изоморфна $\mathbb{P}^{2}$ над полем функций $\mathbb{P}^{1}$, так что и само многообразие $V$ рационально.

При $d=4$, согласно предложению $3.3, V_{\eta}$ всегда перестраивается в расслоение на коники, так что и само $V$ перестраивается в расслоение на коники при помощи линка типа I, а значит, нежестко. В целом, информации о структурах Мори на расслоениях при $d=4$ известно немного, однако в работах В.А. Алексеева [27] и К.А. Шрамова [28] был получен следующий критерий рациональности:

Tеорема 4.2 [27], [28]. Пусть $V / \mathbb{P}^{1}$ - неособое расслоение на поверхности дель Пеццо степени 4. Многообразие $V$ рационально, если и только если топологическая эйлерова характеристика $\chi(V)$ равна $0,-4$ или -8 .

ЗАмЕчАНИЕ. В доказательстве используется тот факт, что после раздутия общего сечения расслоения $V / \mathbb{P}^{1}$ и флопа в некоторых прямых мы получаем стандартное расслоение на коники $U / S$ над линейчатой поверхностью $S$, после чего применяется критерий рациональности для таких расслоений на коники. Однако в работе [27] не рассматривался подробно случай $\chi(V)=-4$, куда, по 
формальным причинам, могло попасть расслоение на коники, бирациональное трехмерной кубике (и потому нерациональное, это единственный специальный случай упомянутого критерия рациональности). В [28] прямыми рассуждениями показано, что при $\chi(V)=-4$ многообразие всегда рационально. Добавим, что необходимости рассматривать “стандартные" (в терминологии работы В. А. Алексеева, имеющие лишь нормальные слои) расслоения на поверхности дель Пеццо степени 4 нет, так как все рассуждения работают при любых типах вырождений слоев.

Пусть теперь $1 \leqslant d \leqslant 3$. Для таких многообразий проблема жесткости, как это будет видно из дальнейшего, уже становится содержательной. В некоторых (нежестких) случаях известен также ответ и на вопрос о рациональности.

Неформальный ответ на вопрос о бирациональной жесткости для расслоений на поверхности дель Пеццо малых степеней (не выше трех) звучит так: чем больше расслоение "закручено по базе", тем оно жестче. Проблема только в том, как измерять эту "закрутку". Топологически "сильнее закручено" значит "имеет больше вырождений", и в этом смысле топологическая эйлерова характеристика (которая, фактически, пересчитывает вырождения) могла бы служить мерилом. Однако на основании имеющихся на данный момент результатов видно, что существуют жесткие и нежесткие расслоения с одной и той же топологической эйлеровой характеристикой.

В процессе исследований проблемы жесткости возникли еще два условия: $K^{2}$ - и $K$-условие [6], [29]. Напомним, что в пространстве $\mathbb{R}^{\rho(X)}$, где $X$ - многообразие из категории Мори и $\rho(X)$ - его число Пикара, можно определить два конуса. Один из них - это классический конус эффективных кривых $\mathbf{N E}(X)$, другой - конус подвижных дивизоров $\mathbf{N M}(X)$. Последний определяется очень просто. По определению дивизор называется подвижным, если содержащая его полная линейная система не имеет базисных компонент. Очевидно, сумма двух подвижных дивизоров - снова подвижный дивизор, таким образом, на пространстве $\operatorname{Pic}(X) \otimes \mathbb{R}$ определен конус $\mathbf{N M}(X)$ как выпуклая оболочка подвижных $\mathbb{R}$-дивизоров.

ОПРЕДЕЛЕНИЕ 4.3. Будем говорить, что расслоение на поверхности дель Пеццо $V / \mathbb{P}^{1}$ удовлетворяет:

$K^{2}$-условию, если $\left(-K_{V}\right)^{2} \notin \operatorname{Int} \mathbf{N E}(V)$,

$K$-условию, если $\left(-K_{V}\right) \notin \operatorname{Int} \mathbf{N M}(V)$.

Эти условия можно переформулировать следующим образом. На $V / \mathbb{P}^{1}$ выполнено $K^{2}$-условие, если при любых $n$ одномерные циклы $n K^{2}-f$ неэффективны, где $f$ - класс прямой в слое расслоения, а $K$-условие эквивалентно тому, что все линейные системы вида $|n(-K)-F|$, где $F$ - класс слоя, либо пусты, либо имеют неподвижную компоненту.

Заметим, что если на расслоении выполнено $K^{2}$-условие, то выполнено и $K$ условие (но не наоборот). Действительно, если линейная система $\left|n\left(-K_{V}\right)-F\right|$ подвижна при некотором $n>0$, то для общих дивизоров $D_{1}$ и $D_{2}$ из этой линейной системы 1 -цикл $D_{1} \circ D_{2} \sim n^{2}\left(-K_{V}\right)^{2}-2 n d f$ будет эффективен.

Вместо $K^{2}$-условия можно было бы рассматривать сдвинутые на некоторое число (скажем, $\alpha$ ) условия вида $K^{2}-\alpha f \notin \operatorname{Int} \mathbf{N E}(V)$. Ни “обычное", ни 
"сдвинутое" $K^{2}$-условие жесткость не "улавливает", как это будет видно из примеров в разделах 5 и 6 , но следует отметить, что "обычное" условие является достаточным для жесткости [6]. На данный момент все надежды связаны с $K$-условием.

ГипотезА 4.4 (основная гипотеза о жесткости). Расслоение $V / \mathbb{P}^{1}$ на поверxности дель Пецио степени $1 \leqslant d \leqslant 3$ бирационально жестко тогда и только тогда, когда для него выполнено $K$-условие.

Основаниями для выдвижения этой гипотезы послужили следующие обстоятельства. Во-первых, для неособых $V$ и степеней 1 и 2 эта гипотеза доказана (в последнем случае при некоторых предположениях общности), подробнее об этом сказано в соответствующих разделах. Затем, во всех встречавшихся на данный момент нежестких случаях она тоже работает. Наконец, есть следующий результат при $d=1$.

Теорема 4.5 [30]. Если расслоение $V / \mathbb{P}^{1}$ на поверхности дель Пецио степени 1 бирационально жестко, то на $V / \mathbb{P}^{1}$ выполнено $K$-условие.

ДокАЗАТЕЛЬство. Предположим обратное, т.е. $V / \mathbb{P}^{1}$ жестко и для $n \gg 0$ существует непустая полная линейная система $\mathscr{D}=\left|n\left(-K_{V}\right)-F\right|$ без неподвижных компонент.

Заметим, что лог-пара $K_{V}+\frac{1}{n} \mathscr{D}$ канонична вдоль любой горизонтальной (накрывающей базу) кривой. Действительно, предположив противное, мы найдем некоторую неприводимую горизонтальную (т.е. накрывающую базу) кривую $B$ такую, что $\nu=$ mult $_{B} \mathscr{D}>n$, и тогда немедленно получим противоречие: индекс пересечения кривой из антиканонической линейной системы общего слоя, не лежащей в базисном множестве $\mathscr{D}$ и пересекающей $B$, с общим элементом $\mathscr{D}$ равен $n$ и не может быть меньше $\nu$.

Итак, мы можем полагать, что $\mathscr{D}$ не имеет неподвижных компонент и пара $K_{V}+\frac{1}{n} \mathscr{D}$ канонична вдоль любой горизонтальной кривой. Запустим программу Саркисова для этой пары с целью сделать ее каноничной всюду (раздел 2). Мы получаем конечную цепочку линков типа II (тип I невозможен по предложению 3.3)

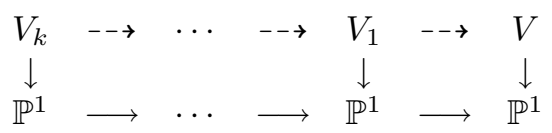

такую, что $K_{V_{k}}+\frac{1}{n} \mathscr{D}_{k}$ канонична, где $\mathscr{D}_{k}-$ собственный прообраз $\mathscr{D}$. Заметим, что в данном случае все элементарные перестройки реализованы следующим образом: вначале мы раздуваем что-то, лежащее в некотором слое, затем делаем несколько лог-флипов над базой, после чего стягиваем собственный прообраз этого слоя. Отсюда видно, что наша цепочка перестроек дает изоморфизм над некоторым открытым подмножеством базы, поэтому $\mathscr{D}_{k} \subset\left|n\left(-K_{V_{k}}\right)+a F\right|$ ( $F$ по-прежнему обозначает класс слоя). Все, что нам нужно, это показать, что $a<0$.

Пусть $V_{k} \leftarrow W \rightarrow V$ - общее разрешение особенностей (“домик Хиронаки"), $C \subset V$ - общая кривая, не задевающая множество неопределенностей морфизма $W \rightarrow V$, и $C_{W}-$ собственный прообраз этой кривой на $W$. Тогда собственный прообраз пары $K_{V}+\frac{1}{n} \mathscr{D}$ пересекается отрицательно с $C_{W}$. Но 
если $a \geqslant 0$, то, ввиду каноничности пары $K_{V_{k}}+\frac{1}{n} \mathscr{D}_{k}$, ее собственный прообраз должен иметь неотрицательное пересечение с $C_{W}$, что противоречит предыдущему.

Итак, $a<0$ и мы можем применить элементарную перестройку типа 3 или 4 к паре $\left(V_{k}, K_{V_{k}}+\frac{1}{n} \mathscr{D}_{k}\right)$. Мы получим либо многообразие Фано (перестройка типа 3), либо расслоение на коники или поверхности дель Пеццо (перестройка типа 4). Любая из полученных структур расслоения Мори будет отлична от $V / \mathbb{P}^{1}$, что противоречит жесткости.

ЗАмечАниЕ. Весьма вероятно, что теорема 4.5 верна и при степени слоя, равной 2 или 3. Для доказательства в этих случаях достаточно показать, что непустые линейные системы вида $|n(-K)-F|$ без неподвижных компонент при $n \gg 0$ не имеют высоких кратностей (больших, чем $n$ ) вдоль горизонтальных кривых.

ЗАмечАниЕ. В случае расслоений на поверхности дель Пеццо степени 1 или 2 с горенштейновыми терминальными особенностями можно в явном виде предъявить бирациональную перестройку на другое расслоение Мори и тем самым доказать теорему 4.5. Конструкция таких расслоений в виде двойных накрытий (разделы 5 и 6) показывает, что из существования непустых линейных систем вида $\left|n\left(-K_{V}\right)-F\right|$ без неподвижных компонент следует существование линейной системы $\left|-K_{V}-F\right|$ с теми же свойствами и с гладким общим элементом. Если $D$ - такой элемент, то из формулы присоединения сразу следует, что $D$ - рациональная эллиптическая поверхность. А тогда мы можем выбрать общий подпучок $\mathscr{P} \subset\left|-K_{V}-F\right|$ и разрешить его базисное множество. На построенном таким образом многообразии собственный прообраз пучка задает морфизм на $\mathbb{P}^{1}$. Слои этого морфизма, очевидно, рациональные поверхности, и остается только применить относительный вариант программы минимальных моделей. Мы получим структуру расслоения на коники или поверхности дель Пеццо, которая будет лежать “поперек" исходной. Указанная процедура, по сути дела, воспроизводит шаги программы Саркисова.

4.3. Перестройки слоя и "хорошие" модели. Под перестройкой слоя расслоения на поверхности дель Пеццо $\rho: V \rightarrow S$ мы понимаем бирациональное над базой отображение $\chi: V / S \rightarrow V^{\prime} / S$ такое, что индуцированное отображение слоев над общей схемной точкой $\eta$ кривой $S$ (которое в общем случае бирационально) является изоморфизмом: $\chi_{\eta}: V_{\eta} \stackrel{\simeq}{\longrightarrow} V_{\eta}^{\prime}$.

Тема перестроек слоя является одной из наиболее важных для понимания бирациональной геометрии расслоений на поверхности дель Пеццо. Действительно, перестройки слоя, очевидно, раскладываются на элементарные перестройки слоя, реализуемые линками типа II. Для доказательства основной гипотезы о жесткости 4.4 достаточно проверить, что $K$-условие сохраняется при элементарных перестройках слоя. Кроме того, эти перестройки, очевидно, "зашиты" в определение 1.2 множества структур Мори, и здесь возникают важные вопросы. Во-первых, сколько представителей содержит класс $[V / S] \in \mathscr{M} \mathscr{S}(V)$ ? Во-вторых, из практических соображений в каждом классе из $\mathscr{M} \mathscr{S}(V)$ нам желательно выбрать такого представителя (так называемую “хорошую модель"), который был бы наиболее удобен с точки зрения описания и работы. 
Сравним ситуацию с двумерной, т.е. с поверхностями, расслоенными над кривой. Для линейчатых поверхностей аналогом является элементарная перестройка слоя: мы раздуваем точку в слое и затем стягиваем собственный прообраз слоя. Отметим, что исходный слой заменяется исключительным дивизором. С другой стороны, для неособых относительно минимальных расслоений на кривые рода 1 и выше верен следующий факт: любое бирациональное над базой отображение между такими поверхностями является, на самом деле, изоморфизмом. Для расслоений на поверхности дель Пеццо малых степеней ситуация, как мы увидим, окажется аналогичной последнему случаю.

Нас будут интересовать, главным образом, расслоения на поверхности дель Пеццо степени $1 \leqslant d \leqslant 3$. Пусть $V / S$ и $U / S$ - такие расслоения и $\chi: V \rightarrow U$ - бирациональное над базой отображение, т.е. мы имеем коммутативную диаграмму

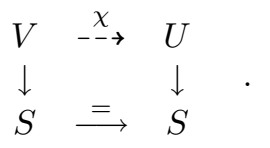

Специализация над общей схемной точкой $\eta$ кривой $S$ дает бирациональное отображение

$$
\chi_{\eta}: V_{\eta} \rightarrow U_{\eta}
$$

Согласно предложению $3.3, \chi_{\eta}$ является изоморфизмом, если $d=1$, композицией инволюций Бертини, если $d=2$, и композицией инволюций Бертини или Гейзера, если $d=3$. Эти бирациональные инволюции можно строить как послойные бирациональные автоморфизмы $V / S$, нетривиально действующие в каждом слое. Но, в любом случае, “открутив" $\chi$ на эти автоморфизмы, мы придем к ситуации, когда у нас есть бирациональное отображение, индуцирующее изоморфизм слоев над общей схемной точкой (и изоморфизм почти всех слоев).

Итак, будем полагать, что $\chi$ составлен из перестроек слоев. Ясно, что если мы выкинем конечное число точек $P_{1}, \ldots, P_{l}$ из $S$, то $\chi$ даст изоморфизм $U$ и $V$ над $S \backslash\left\{P_{1}, \ldots, P_{l}\right\}$. Другими словами, $\chi$ раскладывается в композицию

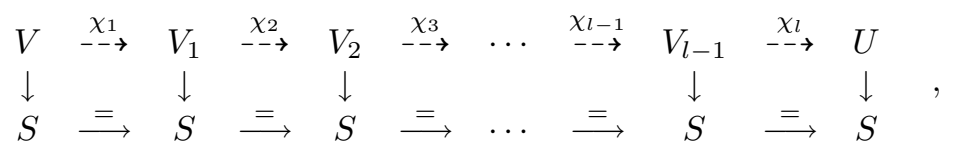

где $\chi_{i}$ - изоморфизм над $S \backslash\left\{P_{i}\right\}$, а все $V_{i} / S$ являются расслоениями Мори.

ТЕОРема 4.6 (единственность неособой модели, [30]-[32]). Пусть $V / S u$ $U / S$ - неособые расслоения на поверхности дель Пещцо степени $1 \leqslant d \leqslant 3$ над кривой $S, \chi$ - биращиональное над базой отображение

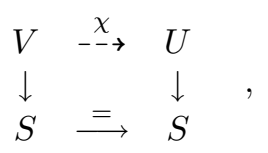

индуцирующее изоморфизм слоев над общей схемной точкой $S$ (m.е. перестройка слоя). Тогда х является изоморфизмом. 
ЗАмечание. Утверждение теоремы, на самом деле, носит локальный характер, т.е. кривая $S$ может быть неполной или даже ростком кривой. Результат для $d=3$ доказан А. В. Пухликовым [32] в гораздо более общей ситуации (для расслоений на гиперповерхности ограниченных степеней в любой размерности). Мы приведем доказательство лишь для степеней 1 и 2, поскольку доказательства в работах [30] и [31] содержат пробелы. Отметим, что в целом все они устроены одинаково: записываем уравнения многообразий и следим за мономами.

ДокАЗАТЕЛЬСТво ДЛЯ СЛУчАЯ $d=1$. Теорему будем доказывать в следующей ситуации. Пусть $\mathscr{R}$ - кольцо дискретного нормирования, $\mathfrak{m}-$ его максимальный идеал, $\mathfrak{m}=t \mathscr{R}$, где $t$ - локальный параметр, $\mathfrak{k}-$ поле функций $\mathscr{R}$. Мы полагаем $S=\operatorname{Spec} \mathscr{R}$ и $\eta=\mathbf{S p e c k}$. Далее, $V$ и $U-$ поверхности дель Пеццо степени 1 , определенные над $\mathscr{R}$. Мы полагаем эти поверхности вложенными в два экземпляра $P$ и $R$ пространства $\mathbb{P}_{\mathscr{R}}(1,1,2,3)$, согласно предложению 3.1 , с координатами соответственно $[x, y, z, w]$ и $[p, q, r, s]$ весов $(1,1,2,3)$. Специализации $P$ и $R$ над $\eta$ обозначим соответственно $P_{\eta}$ и $R_{\eta}$.

По условию, существует изоморфизм $\chi_{\eta}: V_{\eta} \rightarrow U_{\eta}$. Этот изоморфизм индуцирует изоморфизм (мы сохраняем обозначение) $\chi_{\eta}: P_{\eta} \rightarrow R_{\eta}$, что следует из точной тройки

$$
0 \longrightarrow \mathscr{O}_{P_{\eta}}\left(-V_{\eta}\right) \longrightarrow \mathscr{O}_{P_{\eta}} \longrightarrow \mathscr{O}_{V_{\eta}} \longrightarrow 0
$$

подкрученной на $\mathscr{O}(j)$, и вытекающих из нее изоморфизмов

$$
H^{0}\left(V_{\eta}, j\left(-K_{V_{\eta}}\right)\right) \simeq H^{0}\left(P_{\eta}, \mathscr{O}(j)\right)
$$

при $j<6$ (аналогично для $U_{\eta}$ и $R_{\eta}$ ).

Таким образом, мы можем полагать, что поверхности $V$ и $U$, ввиду их неособости, определены как

$$
\begin{aligned}
& V=\left\{w^{2}+z^{3}+z f_{4}(x, y)+f_{6}(x, y)=0\right\} \subset P, \\
& U=\left\{s^{2}+r^{3}+r g_{4}(p, q)+g_{6}(p, q)=0\right\} \subset R,
\end{aligned}
$$

где $f_{i}$ и $g_{i}$ - однородные полиномы соответствующих степеней с коэффициентами в кольце $\mathscr{R}$, а отображения $\chi$ и $\chi^{-1}$ заданы уравнениями

$$
\chi=\left\{\begin{array}{c}
p=t^{\alpha} x \\
q=t^{\beta} y \\
r=t^{\gamma} z \\
s=t^{\delta} w
\end{array}\right\}, \quad \chi^{-1}=\left\{\begin{array}{c}
x=t^{a} p \\
y=t^{b} q \\
z=t^{c} r \\
w=t^{e} s
\end{array}\right\},
$$

Наборы $(\alpha, \beta, \gamma, \delta)$ и $(a, b, c, e)$ состоят из неотрицательных целых чисел, откуда, учитывая веса координат и очевидное соображение $\chi \circ \chi^{-1}=\mathrm{id}$, находим, что для некоторого $m>0$ выполняется

$$
\left\{\begin{array}{rl}
a+\alpha & =m \\
b+\beta & =m \\
c+\gamma & =2 m \\
e+\delta & =3 m \\
2 e & =3 c \\
2 \delta & =3 \gamma
\end{array} .\right.
$$


Последние два равенства получаются, если подставить выражение для $\chi$ из (2) в уравнения (1).

Тогда существуют неотрицательные $k$ и $l$ такие, что $k+l=m$ и

$$
\chi=\left\{\begin{array}{l}
p=t^{\alpha} x \\
q=t^{\beta} y \\
r=t^{2 l} z \\
s=t^{3 l} w
\end{array}\right\}, \quad \chi^{-1}=\left\{\begin{array}{c}
x=t^{a} p \\
y=t^{b} q \\
z=t^{2 k} r \\
w=t^{3 k} s
\end{array}\right\} .
$$

В силу очевидной симметрии по координатам веса 1 полагаем $a \leqslant b$. Пусть

$$
f_{4}=\sum_{i=0}^{4} a_{i} x^{4-i} y^{i}, \quad f_{6}=\sum_{i=0}^{6} a_{i} x^{6-i} y^{i} .
$$

Подставляя теперь (3) в уравнение для $V$ и сокращая на соответствующую степень $t$, находим, что $U$ задано уравнением

$$
s^{2}+r^{3}+r \sum a_{i} t^{4 a+i(b-a)} p^{4-i} q^{i}+\sum b_{i} t^{6 a+i(b-a)} p^{6-i} q^{i}=0 .
$$

Отсюда легко видеть, что если $a>k$, то точка $t=0,(p, q, r, s)=(0: 1: 0: 0)$ на $U$ всегда будет особой.

Предположим теперь, что $a \leqslant k$ и $a \neq 0$. Тогда преобразование для $\chi^{-1}$ в (3) можно заменить на

$$
\chi^{-1}=\left\{\begin{array}{l}
x=p \\
y=t^{b-a} q \\
z=t^{2(k-a)} r \\
w=t^{3(k-a)} s
\end{array}\right\}
$$

так что получаем ситуацию $(a, b, c, d)=(0, b, 2 k, 3 k)$ (для некоторого нового $k$ ). Соответственно, изменится и значение $m$, для которого мы можем полагать, что $b=m$, т.е. $(a, b, c, e)=(0, m, 2 k, 3 k)$, и тогда $(\alpha, \beta, \gamma, \delta)=(0, m, 2 l, 3 l)$. Действительно, если это не так и $b<m$, то $\beta>0$, и тогда

$$
\chi=\left\{\begin{array}{l}
p=t^{m} x \\
q=t^{\beta} y \\
r=t^{2 l} z \\
s=t^{3 l} w
\end{array}\right\}
$$

а далее рассуждаем, как выше: если $\beta>l$, то $V$ имеет особую точку, если же $\beta \leqslant l$, то получаем преобразование

$$
\chi=\left\{\begin{array}{l}
p=t^{m-\beta} x \\
q=t^{y} \\
r=t^{2(l-\beta)} z \\
s=t^{3(l-\beta)} w
\end{array}\right\}
$$


и приходим к набору $(\alpha, \beta, \gamma, \delta)=(0, m, 2 l, 3 l)$ при новом значении $l$ и $m$, и тогда $(a, b, c, e)=(0, m, 2 k, 3 k)$.

Итак, мы можем полагать, что наборы имеют именно такой вид. Так как $k+l=m$, полагаем, что $k \leqslant \frac{m}{2}$ (для $l \leqslant \frac{m}{2}$ рассуждения точно такие же). Подставляя выражение для $\chi^{-1}$ в уравнение $(1)$ для $V$, получим, что $U$ соответствует

$$
s^{2}+r^{3}+r \sum a_{i} t^{m i-4 k} p^{4-i} q^{i}+\sum b_{i} t^{m i-6 k} p^{6-i} q^{i}=0 .
$$

Отметим, что при малых значениях $i$ коэффициенты $a_{i}$ и $b_{i}$ делятся на соответствующую степень $t$. Теперь нетрудно видеть, что точка $t=0,(p, q, r, s)=$ $(0: 1: 0: 0)$ всегда особа на $U$. Таким образом, доказано, что при $d=1$ либо $V$, либо $U$ должна иметь особую точку.

ДокАЗАТЕЛЬСтво для СЛУчАЯ $d=2$. Начало доказательства, с соответствующими изменениями, повторяет предыдущий случай. Полагаем, что $V$ и $U$ заданы полиномами четвертой степени в двух экземплярах $P$ и $R$ пространства $\mathbb{P}_{\mathscr{R}}(1,1,1,2)$. Изоморфизм $\chi_{\eta}: V_{\eta} \rightarrow V_{\eta}$, ввиду изоморфизмов

$$
H^{0}\left(V_{\eta}, j\left(-K_{V_{\eta}}\right)\right) \simeq H^{0}\left(P_{\eta}, \mathscr{O}(j)\right)
$$

при $j<4$, индуцирует изоморфизм $\chi_{\eta}: P_{\eta} \rightarrow R_{\eta}$. Таким образом, существуют координаты $[x, y, z, w]$ и $[p, q, r, s]$ весов $(1,1,1,2)$ такие, что

$$
\chi=\left\{\begin{array}{c}
p=t^{\alpha} x \\
q=t^{\beta} y \\
r=t^{\gamma} z \\
s=t^{\delta} w
\end{array}\right\}, \quad \chi^{-1}=\left\{\begin{array}{c}
x=t^{a} p \\
y=t^{b} q \\
z=t^{c} r \\
w=t^{l} s
\end{array}\right\},
$$

где наборы неотрицательных чисел $(a, b, c, l)$ и $(\alpha, \beta, \gamma, \delta)$ удовлетворяют условию $a+\alpha=b+\beta=c+\gamma=\frac{1}{2}(l+\delta)=m$, и при этом уравнения для $V$ и $U$ могут быть записаны в виде

$$
\begin{aligned}
& V=\left\{w^{2}+\sum_{0 \leqslant i+j \leqslant 4} a_{i j} x^{i} y^{j} z^{4-i-j}=0\right\} \subset P, \\
& U=\left\{s^{2}+\sum_{0 \leqslant i+j \leqslant 4} b_{i j} p^{i} q^{j} r^{4-i-j}=0\right\} \subset R,
\end{aligned}
$$

где коэффициенты $a_{i j}$ и $b_{i j}$ лежат в кольце $\mathscr{R}$.

Предположим сначала, что $a, b, c>\frac{1}{2} l$. Тогда, подставляя выражение для $\chi^{-1}$ из (4) в (5), получаем уравнение для $U$ в виде

$$
U=\left\{s^{2}+\sum_{0 \leqslant i+j \leqslant 4} b_{i j} t^{a i+b j+c(4-i-j)-2 l} p^{i} q^{j} r^{4-i-j}=0\right\},
$$

но так как $a i+b j+c(4-i-j)-2 l>0$, видим, что $U$ имеет особые точки (например, $t=0,[p, q, r, s]=(1: 0: 0: 0))$. 
Итак, учитывая симметрию ситуации в выборе координат веса 1, можем полагать, что $b, c \geqslant a$ и $a \leqslant \frac{1}{2} l$. Но тогда $\chi^{-1}$ можно заменить на эквивалентное преобразование

$$
\chi^{-1}=\left\{\begin{aligned}
x & =p \\
y & =t^{b-a} q \\
z & =t^{c-a} r \\
w & =t^{l-2 a} s
\end{aligned}\right\} .
$$

Переобозначив должным образом числа, полагаем, что $(a, b, c, l)=(0, b, c, l)$. Разумеется, при этом изменится и число $m$.

То же можно сделать и для преобразования $\chi$ : если $\alpha, \beta, \gamma>\frac{1}{2} \delta$, то многообразие $V$ будет особым. Полагая тогда $\alpha, \gamma \geqslant \beta$ и $\beta \leqslant \frac{1}{2} \delta$, мы можем считать, что $(\alpha, \beta, \gamma, \delta)=(\alpha, 0, \gamma, \delta)$. В итоге получаем наборы вида

$$
(a, b, c, l)=(0, m, c, l), \quad(\alpha, \beta, \gamma, \delta)=(m, 0, \gamma, \delta) .
$$

Так как $l+\delta=2 m$, то пусть, например, $l \leqslant m$. Снова, подставляя выражение для $\chi^{-1}$ в (5), находим уравнение для $U$ :

$$
U=\left\{s^{2}+\sum_{0 \leqslant i+j \leqslant 4} b_{i j} t^{m j+c(4-i-j)-2 l} p^{i} q^{j} r^{4-i-j}=0\right\} .
$$

Так как $m j-2 d>0$ при $j \geqslant 3$, то точка $t=0,[p, q, r, s]=(0: 1: 0: 0)$ будет особой на $U$. Действительно, легко проверить, что в открытом подмножестве $\{q \neq 0\}$ пространства $R$ уравнение для $U$ имеет мономы степени не меньше 2 .

Итак, мы показали, что и при $d=1$, и при $d=2$ какое-нибудь из многообразий $V$ или $U$ обязано иметь особую точку. Тем самым теорема 4.6 для этих степеней доказана.

ЗАмечАниЕ. Результат, аналогичный сформулированному выше, был также доказан в [33] для степеней $d \leqslant 4$, используя технику дополнений и принцип связности Шокурова, однако при ограничениях, фактически обесценивающих его: $V$ и $U$ должны иметь невырожденные (неособые) центральные слои, т.е. $V$ и $U$ - это гладкие деформации неособой поверхности дель Пеццо. Расслоения на поверхности дель Пеццо $V / \mathbb{P}^{1}$ малых степеней с такими свойствами не существуют.

В заключение этого раздела приведем несколько примеров перестроек слоя при $d=1$ и $d=2$. Мы придерживаемся обозначений, введенных в соответствующих частях доказательства теоремы 4.6.

ПримеР $(d=1$, “неособый случай"). Пусть $(\alpha, \beta, \gamma, \delta)=(0,6,2,3)$ и $(a, b, c, e)$ $=(6,0,10,15) \cdot V$ и $U$ определены уравнениями

$$
\begin{array}{ll}
V: & w^{2}+z^{3}+x^{5} y+t^{24} x y^{5}=0, \\
U: & s^{2}+r^{3}+p^{5} q+p q^{5}=0 .
\end{array}
$$

Легко проверить, что $U$ неособо, $V$ имеет особую точку типа $c E_{8}$. 
ПримеР ( $d=1$, “бирациональный автоморфизм"). Рассмотрим наборы весов $(\alpha, \beta, \gamma, \delta)=(2,0,2,3)$ и $(a, b, c, e)=(0,2,2,3)$. Уравнения:

$$
\begin{array}{ll}
V: & w^{2}+z^{3}+t^{4} x^{5} y+x y^{5}=0, \\
U: & s^{2}+r^{3}+p^{5} q+t^{4} p q^{5}=0 .
\end{array}
$$

Заметим, что $V$ и $U$ бирегулярно изоморфны: достаточно рассмотреть замену координат $w=s, z=r, x=q, y=p$. Таким образом, мы можем предполагать, что $\chi \in \operatorname{Bir}(X)$ определяется преобразованием в $P_{\eta}$ :

$$
\begin{aligned}
x & \longmapsto t^{-1} y ; \\
y & \longmapsto x ; \\
z & \longmapsto z ; \\
w & \longmapsto w .
\end{aligned}
$$

Очевидно, $V$ имеет $c E_{8}$-особенность в центральном слое.

ПримеР $(d=1$, “ненормальные слои"). Мы приведем два примера с одними и теми же весами $(\alpha, \beta, \gamma, \delta)=(2,0,2,3)$ и $(a, b, c, e)=(0,2,2,3)$ такие, что центральные слои $V_{0}$ и $U_{0}$ (т.е. при $\left.t=0\right)$ ненормальны. Оба случая также будут являться бирациональными автоморфизмами. Первый случай:

$$
\begin{array}{ll}
V: & w^{2}+z^{3}+t z x^{2} y^{2}+t x^{6}+t^{7} y^{6}=0, \\
U: & s^{2}+r^{3}+t r p^{2} q^{2}+t^{7} p^{6}+t q^{6}=0 .
\end{array}
$$

Слой $V_{0}$ ненормален, задается уравнением $w^{2}+z^{3}=0$ ("каспидальный" случай в работе $[22 ;$ п. 1.4$]) . V$ имеет $c E_{7}$-точку в центральном слое.

Второй случай:

$$
\begin{array}{ll}
V: & w^{2}+z^{3}-3 z x^{2} y^{2}+t x^{6}+2 x^{3} y^{3}+t^{7} y^{6}=0, \\
U: & s^{2}+r^{3}-3 r p^{2} q^{2}+t^{7} p^{6}+2 p^{3} q^{3}+t q^{6}=0 .
\end{array}
$$

Центральный слой $V_{0}$ задан как $w^{2}+(z-x y)^{2}(z+2 x y)$ ("нодальный" случай, там же в [22]). Особая точка $V$ имеет тип $c D_{4}$.

ПримеР $(d=2$, “неособый случай"). Пусть $(\alpha, \beta, \gamma, \delta)=(1,4,0,2)$ и $(a, b, c, l)$ $=(3,0,4,6)$, многообразия заданы уравнениями

$$
\begin{array}{ll}
V: & w^{2}+y z^{3}+x^{4}+t^{12} y^{4}=0, \\
U: & s^{2}+q r^{3}+p^{4}+q^{4}=0 .
\end{array}
$$

Отметим, что $U$ неособо, в то же время $V$ имеет $c E_{6}$-точку в $t=0,[x, y, z, w]=$ $(0: 1: 0: 0)$.

ПРимеР $(d=2$, “бирациональный автоморфизм"). Рассмотрим наборы $(\alpha, \beta$, $\gamma, \delta)=(1,2,0,2)$ и $(a, b, c, l)=(1,0,2,2)$, многообразия заданы уравнениями

$$
\begin{array}{ll}
V: & w^{2}+t^{2} z y^{3}+z^{3} y+x^{4}=0, \\
U: & s^{2}+r q^{3}+t^{2} r^{3} q+p^{4}=0 .
\end{array}
$$

Отметим, что замена $p=x, q=z, r=y, s=w$ устанавливает изоморфизм $V$ и $U$. Оба многообразия имеют $c D_{4}$-точку в центральном слое. 


\section{5. Расслоения на поверхности дель Пеццо степени 1}

Пусть $\rho: V \rightarrow \mathbb{P}^{1}$ неособое расслоение на поверхности дель Пеццо степени 1. Согласно предложению 3.1 , каждый слой представляет собой двойное накрытие квадратичного конуса и одновременно поверхность в $\mathbb{P}(1,1,2,3)$, поскольку все слои горенштейновы. Соответственно, есть две конструкции $V / \mathbb{P}^{1}$, являющиеся относительным (над $\mathbb{P}^{1}$ ) вариантом конструкций для слоев. Они, разумеется, однозначно связаны. Основным вариантом для нас будет первый, через двойное накрытие. Таким образом, каждое расслоение, с точностью до деформаций, определяется набором дискретных структурных констант. Два таких набора соответствуют нежестким расслоениям, остальные - жестким.

5.1. Конструкция через двойное накрытие. Итак, пусть $\rho: V \rightarrow \mathbb{P}^{1}-$ неособое расслоение на поверхности дель Пеццо степени $1, \operatorname{Pic} V=\mathbb{Z}\left[-K_{V}\right] \oplus$ $\mathbb{Z}[F], F$ обозначает слой морфизма $\rho$. Очевидно, $\rho_{*} \mathscr{O}\left(-2 K_{V}+m F\right)$ есть локально свободный пучок ранга 4 на $\mathbb{P}^{1}$. Выберем $m$ таким образом, чтобы

$$
\mathscr{E}=\rho_{*} \mathscr{O}\left(-2 K_{V}+m F\right) \simeq \mathscr{O} \oplus \mathscr{O}\left(n_{1}\right) \oplus \mathscr{O}\left(n_{2}\right) \oplus \mathscr{O}\left(n_{3}\right),
$$

где $0 \leqslant n_{1} \leqslant n_{2} \leqslant n_{3}$ - некоторые целые числа; положим $b=n_{1}+n_{2}+n_{3}$.

Заметим, что на $V / \mathbb{P}^{1}$ существует специальное сечение $s_{b}$ : в каждом слое оно высекает базисную точку антиканонического пучка. Формальное его определение такое:

$$
s_{b}=\operatorname{Bas}\left|-K_{V}+k F\right|
$$

при достаточно большом $k$. Напомним, что любой слой $S \in|F|$ неособ в точке $s_{b} \cap S$ (предложение 3.1).

Пусть $\pi: X \rightarrow \mathbb{P}^{1}$ - естественная проекция для $X=\operatorname{Proj} \mathscr{E}, \varphi: V \rightarrow X$ - морфизм, задаваемый линейной системой $\left|-2 K_{V}+m F\right|$ (которая, очевидно, не имеет базисных точек); тогда $\rho=\pi \circ \varphi$. Легко видеть, что если $Q \subset X$ - образ морфизма $\varphi$, то $\varphi: V \rightarrow Q$ - конечный морфизм степени 2 , дивизор ветвления $R_{Q}=R \cap Q$ которого неособ, причем $t_{b} \cap R_{Q}=\varnothing$, где $t_{b}=\varphi\left(s_{b}\right)$ и $R \subset X-$ некоторый дивизор.

На каждый слой $X / \mathbb{P}^{1}$ многообразие $Q$ ограничивается как невырожденный квадратичный конус с вершиной в точке пересечения слоя и $t_{b}$, a $R$ - как кубическая поверхность. Таким образом, если $M$ - тавтологический дивизор на $X$, а $L-$ слой $X / \mathbb{P}^{1}$, то

$$
Q \sim 2 M+a L ; \quad R \sim 3 M+c L .
$$

Пусть $t_{0}$ - сечение $X / \mathbb{P}^{1}$, соответствующее сюръекции $\mathscr{E} \rightarrow \mathscr{O} \rightarrow 0$, и $l-$ класс прямой в слое $L \cong \mathbb{P}^{3}$; положим $t_{b} \sim t_{0}+\varepsilon l$ для некоторого $\varepsilon \geqslant 0$.

Обозначим $H$ очень обильный дивизор вида $M+\beta L, \beta>0$, и $D=H \cap Q$ расслоение на коники с $N=t_{b} \circ H$ вырождениями. Можно считать, что $H$ достаточно общий такой, что все слои $D$ приведены и все особые точки на $D$ дювалевские типа $\mathbb{A}_{1}$.

Легко подсчитать, что $K_{D}^{2}=8-2 b-2 \beta-3 a$. Пусть поверхность $D^{\prime}$ - раздутие всех особых точек и последующее стягивание всех $(-1)$-кривых (а их будет $2 N$ штук). Тогда $D^{\prime}$ - линейчатая поверхность, и мы имеем

$$
K_{D}^{2}+2 N=K_{D^{\prime}}^{2}=8,
$$


откуда

$$
N=b+\beta+\frac{3}{2} a .
$$

Заметим, что $a$ обязано быть четным; положим $a=2 a^{\prime}$. Далее,

$$
N=t_{b} \circ H=\left(t_{0}+\varepsilon l\right) \circ(M+\beta l)=\beta+\varepsilon,
$$

и мы получаем $\varepsilon=b+3 a^{\prime}$. Наконец, так как $R_{Q} \cap t_{b}=\varnothing$, находим, что $0=\left(t_{0}+\varepsilon l\right) \circ(3 M+c L)=3 \varepsilon+c$. Тем самым доказана следующая лемма.

ЛЕмма 5.1. Для $Q, t_{b}$ и $R$ выполнены следующие соотношения:

$$
\begin{aligned}
Q & \sim 2 M-\frac{2}{3}(b-\varepsilon) L, \\
t_{b} & \sim t_{0}+\varepsilon l, \\
R & \sim 3 M-3 \varepsilon L,
\end{aligned}
$$

где $\varepsilon \geqslant 0$ - некоторое иелое число.

В дальнейшем мы всегда полагаем, что $b>0$. При $b=0$, как нетрудно видеть, всегда $Q \sim 2 M$ и $\varepsilon=0$, а тогда $V \cong \mathbb{P}^{1} \times S$, где $S$ - некоторая поверхность дель Пеццо степени 1 , т.е. $V$ рационально, но, главное, не является расслоением Мори ввиду того, что $\operatorname{rk} \operatorname{Pic}\left(V / \mathbb{P}^{1}\right)=9>1$.

ЛЕмма 5.2. Возможсны толъко два случая:

1) $\varepsilon=0,2 n_{2}=n_{1}+n_{3}, n_{1}$ и $n_{3}$ четные; таким образом, в этом случае

$$
Q=2 M-2 n_{2} L, \quad t_{b}=t_{0}, \quad R=3 M
$$

2) $\varepsilon=n_{1}>0, n_{3}=2 n_{2}=6 n_{1}, n_{1}$ четное; отсюда

$$
Q \sim 2 M-2 n_{2} L, \quad t_{b} \sim t_{0}+n_{1} l, \quad R \sim 3 M-3 n_{1} L .
$$

ДокАЗАтельство. Предположим сначала, что $\varepsilon>0$. Тогда $\varepsilon \geqslant n_{1}$ ввиду неприводимости $t_{b}$. Далее, так как $R$ неприводим на $X$, а кривые класса $t_{0}+n_{2} l$ заметают любой эффективный дивизор класса $M-n_{3} L$, то обязательно $\left(t_{0}+n_{2} l\right) \circ R=3 n_{2}-3 \varepsilon \geqslant 0$, т.е. $\varepsilon \leqslant n_{2}$.

Предположим, что $n_{1}=n_{2}=n_{3}$. Так как $t_{b} \circ\left(M-n_{1} L\right)=0$, линейная система $\left|M-n_{1} L\right|$ имеет размерность 2 и каждый ее элемент неприводим, то в ней существует, по крайней мере, одномерное подсемейство, содержащее $t_{b}$. Пусть $C \in\left|M-n_{1} L-t_{b}\right|-$ общий элемент, тогда

$$
Q \circ C=S+S^{\prime} \sim 2 M^{2}-2\left(\frac{1}{3}(b-\varepsilon)+n_{1}\right) M L,
$$

где $S$ и $S^{\prime}$ - неприводимые линейчатые поверхности, накрывающие базу. Заметим, что кривая класса $t_{0}$ одна на $X$ и $t_{b} \cap t_{0}=\varnothing$. Хотя бы одна из указанных поверхностей, скажем, $S$, содержит и $t_{0}$, и $t_{b}$. Легко видеть, что класс $S$ есть $M^{2}-2 n_{1} M L$, откуда $S^{\prime} \sim M^{2}-\frac{4}{3} n_{1} M L$. Поскольку $S \neq S^{\prime}$, то $t_{0} \not \subset S^{\prime}$, и раз $\operatorname{Bas}\left|M-n_{1} L\right|=t_{0}$, то для пересечения $P$ двух общих элементов из $\left|M-n_{1} L\right|$ мы имеем $\operatorname{dim} S^{\prime} \cap P=0$. Таким образом, получаем противоречие:

$$
0 \leqslant S^{\prime} \circ P=\left(M^{2}-\frac{4}{3} n_{1} M L\right) \circ\left(M^{2}-2 n_{1} M L\right)=-\frac{n_{1}}{3}<0 .
$$


Итак, если $\varepsilon \geqslant n_{1}$, то $n_{1}<n_{3}$; кроме того, тогда $n_{1}>0$, так как иначе $R$ содержал бы любую кривую класса $t_{0}$, а таких, как минимум, одномерное семейство, если $n_{1}=0$.

Пусть $G$ - неприводимая линейчатая поверхность, являющаяся пересечением общих элементов из $\left|M-n_{2} L\right|$ и $\left|M-n_{3} L\right|$. Покажем, что $t_{b} \subset G$. Легко видеть, что $G$ есть поверхность класса $\mathbb{F}_{n_{1}}$ и $t_{0}$ - ее исключительное сечение. Если $t_{b} \not \subset G$, то для общего слоя $S \in|L|$ прямая $\left.G\right|_{S}$ не лежит на $\left.Q\right|_{S}$. Таким образом, $G \not \subset Q$, и мы можем считать, что $G \circ Q=t_{0}+C$, где $C$ некоторая кривая, никакая компонента которой не содержит $t_{0}$. Нетрудно подсчитать, что

$$
C \sim t_{0}+2\left(b-n_{2}-n_{3}-\frac{b-\varepsilon}{3}\right) l
$$

откуда

$$
2\left(b-n_{2}-n_{3}-\frac{b-\varepsilon}{3}\right) \geqslant n_{1},
$$

что, учитывая неравенство $\varepsilon \leqslant n_{2}$, приводит к противоречию: $n_{1}>\frac{4}{3} n_{1}$.

Итак, $t_{b} \subset G$. Отсюда, кстати, следует, что $n_{2}<n_{3}$, так как в противном случае $\operatorname{dim}\left|M-n_{3} L\right|=1$ и, следовательно, $\operatorname{Bas}\left|M-n_{3} L\right|=t_{0}$, и мы всегда бы нашли такую $G$, что $t_{b} \not \subset G$.

Обозначим единственный эффективный элемент $\left|M-n_{3} L\right|$ через $T$ и покажем, что $\left.T\right|_{Q}=2 G$. Пусть это не так и $\left.T\right|_{Q}=G+G^{\prime}, G^{\prime} \neq G$. Поскольку $t_{b} \subset G \cap G^{\prime}$ и $t_{0} \subset G$, то $t_{0} \not \subset G^{\prime}$. Тогда

$$
\left.G^{\prime}\right|_{G}=t_{0}+\text { слои в количестве } t_{0} \cap t_{b}=\varepsilon-n_{1} \text {. }
$$

Выберем общий элемент $S \in\left|M-n_{1} L\right|$; ясно, что $\left.S\right|_{T}-$ поверхность класса $\mathbb{F}_{n_{2}}$ с исключительным сечением $t_{0}$, причем $\left.G \cap S\right|_{T}=t_{0}$. Пусть $C=\left.G^{\prime} \cap S\right|_{T}-$ неприводимая кривая, $C \circ t_{0}=\varepsilon-n_{1}$. Имеем $Q \circ T \circ S=t_{0}+C$, откуда

$$
C \sim t_{0}+2\left(n_{2}-\frac{b-\varepsilon}{3}\right) l,
$$

и тогда $C \circ t_{0}=n_{2}-\frac{2}{3}(b-\varepsilon)=\varepsilon-n_{1}$. Получили противоречие:

$$
\varepsilon=n_{1}+n_{2}-2 n_{3}<0 .
$$

Таким образом,

$$
T \circ Q=2 M^{2}-2\left(n_{3}+\frac{b-\varepsilon}{3}\right) M L=2 G=2\left(M^{2}-\left(n_{2}+n_{3}\right) M L\right),
$$

поэтому $2 n_{2}=n_{1}+n_{3}-\varepsilon$. Если $\varepsilon>n_{1}$, то $R_{Q}=\left.R\right|_{Q}$ будет содержать $G$, так как $G$ заметается кривыми класса $t_{0}+n_{1} l$. Значит, $\varepsilon=n_{1}$ и $2 n_{2}=n_{3}$.

Покажем, что $n_{1}$ четно. Пусть $C \sim t_{0}+n_{1} l$ - общая неприводимая кривая на $G$ и $C_{V}$ - ее прообраз на $V$. Тогда $C_{V}$ дважды накрывает $C$ с ветвлением в дивизоре степени $t_{b} \circ C=n_{1}$, которая должна быть четной.

По условию, дивизор $R_{Q}=\left.R\right|_{Q}$ неособ всюду, а значит, и вдоль $t_{0}$. Так как $\left.Q\right|_{T}=2 G$, то $T$ является касательным пространством к $Q$, поэтому $R$ и $T$ нигде 
не касаются вдоль $t_{0}$, так что $R_{T}=\left.R\right|_{T}$ неособо вдоль этого сечения и имеет касательным пространством $G \subset T$. Отсюда немедленно следует, что $n_{2}=3 n_{1}$. Чтобы убедиться в этом, достаточно раздуть сечение $t_{0}$ на

$$
T \cong \operatorname{Proj}_{\mathbb{P}^{1}}\left(\mathscr{O} \oplus \mathscr{O}\left(n_{1}\right) \oplus \mathscr{O}\left(n_{2}\right)\right),
$$

на исключительном дивизоре собственные прообразы $G$ и $R_{T}$ должны высекать одно и то же сечение.

Итак, случай $\varepsilon>0$ полностью разобран. Пусть теперь $\varepsilon=0$. Мы можем считать, что $n_{1}<n_{3}$ (случай $n_{1}=n_{3}$ был разобран выше).

Пусть $T \in\left|M-n_{3} L\right|$. Заметим, что $T=\operatorname{Proj} \mathscr{E}_{T}$, где

$$
\mathscr{E}_{T}=\mathscr{O} \oplus \mathscr{O}\left(n_{1}\right) \oplus \mathscr{O}\left(n_{2}\right) .
$$

Так как $T$ заметается кривыми класса $t_{0}+n_{2} l$, то из неприводимости $Q \sim$ $2 M-\frac{2}{3} b L$ следует, что $n_{2} \geqslant \frac{b}{3}$, т.е. $2 n_{2} \geqslant n_{1}+n_{3}$, и раз $n_{3}>n_{1}$, то $n_{2}>n_{1}$.

Далее, пусть $C \sim t_{0}+n_{1} l$ неприводима; $C \circ Q=2\left(n_{1}-\frac{b}{3}\right)<0$, так как $b=n_{1}+n_{2}+n_{3}>3 n_{1}$. Значит, $C \subset Q$. Линейчатая поверхность $G \sim\left(M-n_{2} L\right) \circ$ $\left(M-n_{3} L\right)=M^{2}-\left(n_{2}+n_{3}\right) M L$ имеет класс $\mathbb{F}_{n_{1}}$ и заметается кривыми того же класса, что и $C$, поэтому $G \subset Q$. Таким образом, $\left.T\right|_{Q}=G+G^{\prime}$.

Пусть $M_{T}=\left.M\right|_{T}$ - тавтологический дивизор на $T, L_{T}=\left.L\right|_{T}$. Тогда в $T$ класс $G$ есть $M_{T}-n_{2} L_{T},\left.Q\right|_{T} \sim 2 M_{T}-\frac{2}{3} b L_{T}$, откуда $G^{\prime} \sim M_{T}-\left(\frac{2}{3} b-n_{2}\right) L_{T}$. Заметим, что $\frac{2}{3} b-n_{2}>n_{1}$, поскольку $n_{1}<n_{2}$.

Однако в $T$ только один неприводимый дивизор класса $M_{T}-x L_{T}$ при $x>n_{1}$ : это $M_{T}-n_{2} L_{T}$. Значит, $\frac{2}{3} b-n_{2}=n_{2}$, откуда $n_{1}+n_{3}=2 n_{2}$.

Осталось показать, что $n_{1}$ и $n_{3}$ четные. Пусть $C \subset Q$ - общая неприводимая кривая класса $t_{0}+n_{1} l, C_{V}$ - ее прообраз на $V$. Тогда $C_{V}$ есть двойное накрытие $C$ с ветвлением в дивизоре степени $R \circ C=3 n_{1}$, откуда следует, что $n_{1}$, а значит, и $n_{3}$ четно. Лемма 5.2 доказана.

Лемма 5.3. Неособые расслоения с указанными выше наборами структурных констант $\left(\varepsilon, n_{1}, n_{2}, n_{3}\right)$ существуют.

ДокАЗАтельство. Первым рассмотрим случай структурного набора $\left(\varepsilon, n_{1}\right.$, $\left.n_{2}, n_{3}\right)=\left(n_{1}, n_{1}, 3 n_{1}, 6 n_{1}\right)$, где $n_{1}>0$ - четное число. Сначала в предположении о том, что существует расслоение на невырожденные квадратичные конусы $Q \subset \operatorname{Proj} \mathscr{E}$, покажем, что найдется дивизор $R \in\left|3 M-3 n_{1} L\right|$ на $X$ такой, что ограничение $R_{Q}=\left.R\right|_{Q}$ неособо. Воспользуемся обозначениями из предыдущей леммы. Напомним, что $T$ - единственный элемент линейной системы $\left|M-n_{3} L\right|$ и

$$
\mathscr{E}_{T}=\mathscr{O} \oplus \mathscr{O}\left(n_{1}\right) \oplus \mathscr{O}\left(n_{2}\right) .
$$

Заметим, что любой элемент из $\left|3 M_{T}-3 n_{1} L_{T}\right|$ поднимается до элемента из $\left|3 M-3 n_{1} L\right|$, где $M_{T}=\left.M\right|_{T}$ и $L_{T}=\left.L\right|_{T}$ - соответственно тавтологический дивизор и слой на $T$. Кроме того, дивизор $G+K \in\left|3 M_{T}-3 n_{1} L_{T}\right|$ неособ вдоль сечения $t_{0}$, где $K \in\left|2 M_{T}\right|$ - общий элемент ( $K$ не содержит $t_{0}$, так как $M_{T}$ ○ $\left.t_{0}=0\right)$. Таким образом, поскольку $\left.Q\right|_{T}=2 G$, то общий элемент $R \in \mid 3 M-$ $3 n_{1} L \mid$ и его ограничение $R_{Q}=\left.R\right|_{Q}$ неособы вдоль $t_{0}$, а раз линейная система $\left|3 M-3 n_{1} L\right|$ и ее ограничение на $Q$ имеют базисным множеством само $t_{0}$, то из теоремы Бертини следует, что общий элемент $R_{Q}$ неособ на $Q$. 
Теперь покажем, что расслоение $Q \sim 2 M-2 n_{2} L$ на невырожденные конусы существует. Заметим, что если это так, то, раздув кривую особенностей $t_{b} \subset Q$, мы получим $\mathbb{P}^{1}$-расслоение $\widetilde{Q}$ над линейчатой поверхностью $B$, являющееся проективизацией некоторого локально свободного пучка $\mathscr{E}_{B}$ ранга 2 на $B$. Заметим, что этот пучок должен быть разложимым, поскольку $\widetilde{Q}$ будет иметь два непересекающихся сечения: исключительный дивизор раздутия и прообраз какого-нибудь неприводимого элемента из $\left|\left(M-n_{1} L\right)\right|_{Q} \mid$. Мы решим нашу задачу, если предъявим расслоение с требуемыми параметрами.

Пусть $B$ - линейчатая поверхность класса $\mathbb{F}_{3 n_{1}}, s_{B}$ и $l_{B}$ - ее минимальное сечение и слой. Положим

$$
\mathscr{E}_{B}=\mathscr{O}_{B} \oplus \mathscr{O}_{B}\left(2 s_{B}+5 n_{1} l_{B}\right)
$$

и рассмотрим проекцию

$$
\pi: \widetilde{Q}=\operatorname{Proj} \mathscr{E}_{B} \longrightarrow B .
$$

Минимальное сечение расслоения $\widetilde{Q} \rightarrow B$, т.е. сечение, соответствующее проекции $\mathscr{E}_{B} \rightarrow \mathscr{O}_{B} \rightarrow 0$, обозначим также через $B$. Пусть $\widetilde{G}=\pi^{-1}\left(s_{B}\right)$ и $\widetilde{L}=\pi^{-1}\left(l_{B}\right)$. Очевидно,

$$
\operatorname{Pic}(\widetilde{Q})=\mathbb{Z}[\widetilde{G}] \oplus \mathbb{Z}[\widetilde{L}] \oplus \mathbb{Z}[B],
$$

причем линейчатая поверхность $\widetilde{G}$ имеет класс $\mathbb{F}_{n_{1}}$ и ее минимальное сечение $\widetilde{t}_{0}$ не пересекает $B$, а поверхности из $|\widetilde{L}|$ имеют класс $\mathbb{F}_{2}$. Заметим также, что неприводимые элементы из $\left|B+2 \widetilde{G}+5 n_{1} \widetilde{L}\right|$ суть сечения морфизма $\pi$, не пересекающие $B$.

Рассмотрим обратимый пучок $\widetilde{\mathscr{M}}=\mathscr{O}_{B}\left(B+2 \widetilde{G}+6 n_{1} \widetilde{L}\right)$, естественную проекцию $\mu: B \rightarrow \mathbb{P}^{1}$, композицию $\varphi=\pi \circ \mu: \widetilde{Q} \rightarrow \mathbb{P}^{1}$, и пусть $\mathscr{E}=\varphi_{*} \widetilde{\mathscr{M}}$. Легко видеть, что

$$
\pi_{*} \widetilde{\mathscr{M}}=\mathscr{E}_{B} \otimes \mathscr{O}_{B}\left(n_{1} l_{B}\right)=\mathscr{O}_{B}\left(n_{1} l_{B}\right) \oplus \mathscr{O}_{B}\left(2 s_{B}+6 n_{1} l_{B}\right),
$$

а $\rho_{*} \mathscr{O}_{B}\left(2 s_{B}\right)=\mathscr{O}\left(-6 n_{1}\right) \oplus \mathscr{O}\left(-3 n_{1}\right) \oplus \mathscr{O}$, так как поверхность $B$ имеет класс $\mathbb{F}_{3 n_{1}}$. Таким образом, находим

$$
\mathscr{E}=\mathscr{O} \oplus \mathscr{O}\left(n_{1}\right) \oplus \mathscr{O}\left(3 n_{1}\right) \oplus \mathscr{O}\left(6 n_{1}\right) .
$$

Обозначим $X=\operatorname{Proj} \mathscr{E}$ и $M$ тавтологический дивизор на $X$. Теперь легко видеть, что линейная система $\left|B+2 \widetilde{G}+6 n_{1} \widetilde{L}\right|$ задает послойный бирациональный морфизм

$$
\alpha: \widetilde{Q} \rightarrow Q \subset X,
$$

стягивающий линейчатую поверхность $B$ на ее образующую, причем $\alpha_{*} \widetilde{M}=$ $\mathscr{O}_{Q}\left(\left.M\right|_{Q}\right)$, а $Q$ является расслоением на квадратичные конусы с требуемыми свойствами. Отметим, что образом $\widetilde{t}_{0}$ будет сечение $t_{0}$ в $X$, соответствующее проекции $\mathscr{E} \rightarrow \mathscr{O} \rightarrow 0$, а линия вершин конусов в $Q$ будет сечением класса $t_{0}+n_{1} l$, где $l-$ класс прямой в слое $X \rightarrow \mathbb{P}^{1}$.

Пусть теперь $\left(\varepsilon, n_{1}, n_{2}, n_{3}\right)=\left(0, n_{1}, \frac{1}{2}\left(n_{1}+n_{3}\right), n_{3}\right)$. Существование неособого дивизора ветвления $R_{Q}=\left.R\right|_{Q}$ немедленно следует из теоремы Бертини, поскольку линейная система $|3 M|$ не имеет базисных точек и ее общий элемент 
не пересекает кривую особенностей $Q$. Чтобы показать, что многообразие $Q$ с требуемыми свойствами существует, рассмотрим линейчатую поверхность $B$ класса $\mathbb{F}_{n_{3}-n_{1}}$ с минимальным сечением $s_{B}$ и слоем $l_{B}$, а на ней расслоение ранга 2

$$
\mathscr{E}_{B}=\mathscr{O}_{B} \oplus \mathscr{O}_{B}\left(2 s_{B}+n_{3} l_{B}\right),
$$

и далее действуем в точности так, как выше. Лемма 5.3 доказана.

Итак, мы показали, что неособые расслоения на поверхности дель Пеццо степени 1, с точностью до деформаций, образуют две серии, зависящие от одного и от двух дискретных параметров, и при любых допустимых значениях параметров реализуются:

- специальная серия: $\left(\varepsilon, n_{1}, n_{2}, n_{3}\right)=\left(n_{1}, n_{1}, 3 n_{1}, 6 n_{1}\right), n_{1}>0$ - четное число;

- основная серия: $\left(\varepsilon, n_{1}, n_{2}, n_{3}\right)=\left(0, n_{1}, \frac{1}{2}\left(n_{1}+n_{3}\right), n_{3}\right), n_{1}$ и $n_{3}$ - неотрицательные четные числа.

Конструкция $V$ как двойного накрытия $Q$ позволяет легко вычислить пересечения циклов на $V$. Обозначим $G_{V}$ прообраз поверхности $G \sim\left(M-n_{2} L\right)$ 。 $\left(M-n_{3} L\right)$ на $V$, через $F-$ то же для $L_{Q}=\left.L\right|_{Q}$ и через $H-$ для $M_{Q}=\left.M\right|_{Q}$. Пусть также $2 s_{0}$ - прообраз $t_{0}$, а $2 f$ - прообраз прямой в слое $L_{Q}$.

Лемма 5.4. $\overline{\mathbf{N E}}(V)=\mathbf{N E}(V)=\mathbb{R}_{+}\left[s_{0}\right] \oplus \mathbb{R}_{+}[f]$, при этом:

1) для основной серии

$$
\begin{gathered}
K_{V} \sim-G_{V}+\left(\frac{1}{2} n_{1}-2\right) F, \quad H \sim 2 G_{V}+n_{3} F \\
K_{V}^{2} \sim s_{0}+\left(4-n_{2}\right) f, \quad s_{b} \sim s_{0}, \quad s_{0} \circ F=f \circ G_{V}=1, \\
s_{0} \circ G_{V}=-\frac{1}{2} n_{3}, \quad f \circ F=0 ;
\end{gathered}
$$

2) для специальной серии

$$
\begin{gathered}
K_{V} \sim-G_{V}-\left(\frac{1}{2} n_{1}+2\right) F, \quad H \sim 2\left(G_{V}+n_{2} F\right), \quad s_{b} \sim s_{0}+\frac{1}{2} n_{1} f, \\
K_{V}^{2} \sim s_{0}+\left(4+\frac{3}{2} n_{1}-n_{2}\right) f, \quad s_{0} \circ F=f \circ G_{V}=1, \\
s_{0} \circ G_{V}=-\frac{1}{2} n_{3}=-n_{2}, \quad f \circ F=0 .
\end{gathered}
$$

5.2. Конструкция через вложение в $\mathbb{P}_{\mathbb{P}^{1}}(1,1,2,3)$. Как уже отмечалось, неособые (на самом деле, горенштейновы) расслоения на поверхности дель Пеццо степени 1 можно строить как гиперповерхности в расслоениях на взвешенные проективные пространства. Следующая конструкция извлечена из [34].

Пусть $\mathscr{S}=\bigoplus_{n \geqslant 0} \mathscr{S}_{n}$ - пучок градуированных алгебр на $\mathbb{P}^{1}$ со свойствами:

(a) $\mathscr{S}_{1}=\mathscr{E}=\mathscr{O} \oplus \mathscr{O}(a)$, где $a \geqslant 0$;

(b) $\mathscr{S}_{2}=S^{2} \mathscr{E} \oplus \mathscr{F}$, где $\mathscr{F}=\mathscr{O}(b)$;

(c) $\mathscr{S}_{3}=S^{3} \mathscr{E} \oplus \mathscr{E} \otimes \mathscr{F} \oplus \mathscr{G}$, где $\mathscr{G}=\mathscr{O}(c)$;

(d) $\mathscr{S}$ порождена $\mathscr{E}, \mathscr{F}$ и $\mathscr{G}$. 
Рассмотрим расслоение $\pi: X=\operatorname{Proj} \mathscr{S} \rightarrow \mathbb{P}^{1}$ на взвешенные проективные пространства с весами $(1,1,2,3)$. Пусть $H$ - тавтологический $\mathbb{Q}$-Картье дивизор, другими словами, $6 H$ - дивизор Картье, $\mathscr{O}_{X}(H)$ - рефлексивный пучок ранга 1 такой, что $\pi_{*} \mathscr{O}_{X}(H)=\mathscr{S}_{1}, \pi_{*} \mathscr{O}_{X}(2 H)=\mathscr{S}_{2}, \pi_{*} \mathscr{O}_{X}(3 H)=\mathscr{S}_{3}$, и пусть $F-$ слой $X$. Мы полагаем, что наше неособое расслоение $V \rightarrow \mathbb{P}^{1}$ на поверхности дель Пеццо степени 1 является элементом линейной системы $V \in|6 H-k F|$ для некоторого целого $k$.

Пусть $\mathscr{M}$ - подалгебра в $\mathscr{S}$, порожденная $\mathscr{F}$ и $\mathscr{G}, T=\operatorname{Proj} \mathscr{M} \subset X$ соответствующая линейчатая поверхность класса $\mathbb{F}|3 b-2 c|$, а $s_{1}$ и $s_{2}$ - сечения $X / \mathbb{P}^{1}$, отвечающие проекциям $\mathscr{S} \rightarrow S(\mathscr{F})$ и $\mathscr{S} \rightarrow S(\mathscr{G})$. Очевидно, $X$ имеет фактор-особенности типа $\frac{1}{2}(1,1,1)$ вдоль $s_{1}$ и $\frac{1}{3}(1,1,2)$ вдоль $s_{2}$. Отметим, что сечения $s_{1}$ и $s_{2}$ на $T$ не пересекаются. Так как $V$ неособо, сечения $s_{1}$ и $s_{2}$ не пересекают $V$. Отсюда следует, что $T \not \subset V$, т.е. кривая $\left.V\right|_{T}$ эффективна и нигде не пересекает $s_{1}$ и $s_{2}$. Это возможно только на линейчатых поверхностях класса $\mathbb{F}_{0}$, отсюда $3 b=2 c$. Кроме того, если $f-$ слой поверхности $T$, то $\left.V\right|_{T} \sim s_{2}+(2 c-k) f$, откуда $k=2 c=3 b$. Итак, мы нашли все необходимые связи между параметрами.

Для того чтобы сравнить их с набором $\left(\varepsilon, n_{1}, n_{2}, n_{3}\right)$, достаточно найти $\rho_{*}\left(-K_{V}\right)$ и $\rho_{*}\left(-2 K_{V}\right)($ и, возможно, подкрутить прямые образы) в конструкциях двойного накрытия. Мы получим следующий результат.

ПреДЛОЖенИЕ 5.5. Параметры $(a, b, c, k)$ в конструкиии $V \subset X=\operatorname{Proj} \mathscr{S}$ удовлетворяют соотношениям $a \geqslant 0 u 3 b=2 c=k$. Их связь с набором $\left(\varepsilon, n_{1}, n_{2}, n_{3}\right)$ определяется следующими равенствами:

(a) специальная серия: $a=3 n_{1}, b=n_{1}, c=\frac{3}{2} n_{1}, k=3 n_{1}$;

(b) основная серия: $a=\frac{1}{2}\left(n_{3}-n_{1}\right), b=-n_{1}, c=-\frac{3}{2} n_{1}, k=-3 n_{1}$.

\section{3. Бирационально жесткие расслоения.}

Теорема 5.6. Гипотеза 4.4 верна для неособых расслоений Мори на поверхности дель Пецио степени 1. Бирационально жесткими будут все расслоения основной и специальной серий, чей набор структурных констант $\varepsilon, n_{1}, n_{2}, n_{3}$ отличен от $(0,2,2,2)$ u $(0,0,1,2)$ (основная серия). При этом бирачионально жесткие расслоения являются единственными неособыми расслоениями Мори в своем классе бирациональной эквивалентности, их группь бирациональных автоморфизмов конечны и совпадают с группами бирегулярных автоморфизмов, которые в общем случае порождены инволюиией двойного накрытия.

Как видно из этой теоремы и леммы 5.4, существуют бирационально жесткие расслоения, удовлетворяющие $K$-условию, но не удовлетворяющие $K^{2}$-условию. В этих случаях структурные наборы $\left(\varepsilon, n_{1}, n_{2}, n_{3}\right)$ следующие: специальная серия - $(2,2,6,12)$, основная серия - $(0,0,2,4),(0,0,3,6)$ и $(0,2,3,4)$.

НАБРОСОК ДОКАЗАТЕЛЬСТВА ТЕОРЕМЫ. Вся техническая часть необходимой работы содержится в следующем предложении, доказательство которого мы опустим.

ПредлОЖЕНИЕ 5.7 ([6], с дополнением в [29], предложение 2.7). Пусть V/P 1 - неособое расслоение на поверхности дель Пеццо степени $1, V^{\prime} / S^{\prime}$ - некоторое расслоение Мори, $\chi: V \rightarrow V^{\prime}-$ бирациональное отображсене. Пусть

$$
\mathscr{D}^{\prime}=\mid n^{\prime}\left(-K_{V^{\prime}}\right)+\text { поднятый с базы обильный дивизор } \mid
$$


- очень обильная линейная система. Предположим, что

$$
\mathscr{D}=\chi^{-1} \mathscr{D}^{\prime} \subset\left|n\left(-K_{V}\right)+m F\right|
$$

для некоторого $m \geqslant 0$. Тогда $n=n^{\prime}$ и $\chi$ бирационально над базой.

Предположим сначала, что $\varepsilon=n_{1}>0$ (специальный случай) и линейная система $\mathscr{D} \subset\left|n\left(-K_{V}\right)+m F\right|$ не имеет неподвижных компонент. Тогда для общего элемента $D \in \mathscr{D}$ кривая

$$
\left.D\right|_{G_{V}} \sim n s_{0}+\left[m+n\left(n_{1}-n_{2}+2\right)\right] f
$$

эффективна, так что $2+n_{1}-n_{2}+\frac{m}{n} \geqslant 0$. Но $n_{2}=3 n_{1}$ и $n_{1}>0$ в этом случае, поэтому $m>0$. Нужный результат немедленно следует из предложения 5.7.

Пусть $\varepsilon=0$ (основная серия). Покажем, что если $\mathscr{D} \subset\left|n\left(-K_{V}\right)-m F\right|$ не имеет неподвижных компонент при $m>0$, то обязательно $n_{3}=2$. Действительно, выберем общий элемент $D \in\left|n\left(-K_{V}\right)-m F\right|$. Так как

$$
\left.D\right|_{G_{V}} \sim n s_{0}-\left(m+n\left(\frac{n_{1}}{2}+n_{3}-n_{2}-2\right)\right) f
$$

- эффективная кривая, то

$$
0<\frac{m}{n} \leqslant 2+n_{2}-n_{3}-\frac{n_{1}}{2}=2-\frac{n_{3}}{2} .
$$

Остается учесть, что $n_{3}$ положительно и четно. Таким образом, такого сорта линейные системы могут быть только при структурных константах $(0,2,2,2)$ и $(0,0,1,2)$. Во всех остальных случаях работает предложение 5.7.

Осталось учесть утверждения теорем 4.5 и 4.6, и мы получаем утверждение теоремы 5.6 о бирациональной жесткости, единственности неособой модели и совпадении групп бирациональных и бирегулярных автоморфизмов. Конечность группы последних вытекает из конечности группы бирегулярных автоморфизмов слоя над общей схемной точкой, в которой всегда присутствует инволюция двойного накрытия. Теорема 5.6 доказана.

5.4. Нежесткие случаи. В доказательстве теоремы 5.6 было показано, что при $n_{3}=2$ не выполнено $K$-условие для расслоений основной серии, и по теореме 4.5 они должны иметь несколько структур Мори. Начнем со случая $(0,2,2,2)$.

Теорема 5.8. Пусть $V / \mathbb{P}^{1}$ - неособое расслоение на поверхности дель Пеццо степени 1 со структурными константами $\varepsilon=0, n_{1}=2, n_{2}=2, n_{3}=2$. Существует бирациональное отображение $\psi: V \rightarrow U$ на неособое расслоение $U / \mathbb{P}^{1}$ на поверхности дель Пеццо степени 1 с теми же структурными константами. Отображение $\psi$ является флопом с иентром в сечении $s_{b}=s_{0}$. Множество структур Мори представлено классами расслоении $V / \mathbb{P}^{1}$ и $U / \mathbb{P}^{1}$, и это единственные неособые расслоения Мори в их классе бирациональной эквивалентности; в частности, $V$ нерационально. Группа бирациональньх автоморфизмов конечна и, в общем случае, совпадает с группой бирегулярных автоморфизмов, которал, в свою очередь, в общем случае порождена инволюицей двойного накрытия. 
ДоказАтельство. Заметим, что $\operatorname{dim}\left|\left(-K_{V}\right)-F\right|=1$. Действительно, имеем $G_{V} \sim\left(-K_{V}\right)-F$ и

$$
2 \operatorname{dim}\left|G_{V}\right|=2 \operatorname{dim}|G| \leqslant \operatorname{dim}|2 G|=\operatorname{dim}|(M-2 L)|_{Q} \mid=2 .
$$

Более того, $s_{0}=s_{b}=\operatorname{Bas}\left|\left(-K_{V}\right)-F\right|$, линейная система $\left|n\left(-K_{V}\right)\right|$ не имеет базисных точек при $n \geqslant 2$ и при $n \geqslant 3$ осуществляет малое стягивание $s_{b}$. Сечение $s_{b}$ является полным пересечением любых двух элементов из $\left|\left(-K_{V}\right)-F\right|$; используя лемму 5.4 , находим $\mathscr{N}_{s_{b} \mid V} \simeq \mathscr{O}(-1) \oplus \mathscr{O}(-1)$. Таким образом, существует флоп $\psi: V \rightarrow U$ с центром в $s_{b}$. Неособое многообразие $U$ имеет структуру расслоения на поверхности дель Пеццо степени 1. Действительно, рассмотрим общий элемент $S \in\left|\left(-K_{V}\right)-F\right|$. Используя формулу присоединения, получаем, что $S$ - рациональная эллиптическая поверхность. Флоп $\psi$ стягивает на $S$ сечение (т.е. $(-1)$-кривую) $s_{b}$, так что образом $S$ будет поверхность дель Пеццо степени 1. Отметим, что флоп разведет элементы $\left|\left(-K_{V}\right)-F\right|$, так что имеем морфизм $U \rightarrow \mathbb{P}^{1}$ со слоями - поверхностями дель Пеццо степени 1. Так как $U$ неособо и $\rho(U)=\rho(V)=2$, видим, что $U / \mathbb{P}^{1}$ является расслоением Мори. Положим

$$
\operatorname{Pic}(U)=\mathbb{Z}\left[-K_{U}\right] \oplus \mathbb{Z}\left[F_{U}\right],
$$

где $F_{U}$ обозначает слой $U / \mathbb{P}^{1}$. Поскольку пучок $\left|\left(-K_{V}\right)-F\right|$ при флопе переходит в пучок $F_{U}$, легко подсчитать действие $\psi$ на дивизорах: если $D \sim$ $n\left(-K_{V}\right)+m F$, то

$$
\psi_{*}(D) \sim(n+m)\left(-K_{U}\right)-m F .
$$

Так как $V / \mathbb{P}^{1}$ бирационально нежестко, то расслоение $U / \mathbb{P}^{1}$ может иметь структурные константы либо $\varepsilon=0, n_{1}=2, n_{2}=2, n_{3}=2$, либо $\varepsilon=0, n_{1}=0, n_{2}=1$, $n_{3}=2$. Последний случай невозможен, так как иначе должен был бы существовать эффективный дивизор $G_{U} \sim\left(-K_{U}\right)-2 F_{U}$, что на $U$ невозможно ввиду формулы (6). Таким образом, $U / \mathbb{P}^{1}$ имеет тот же набор структурных констант, что и $V / \mathbb{P}^{1}$.

Рассмотрим теперь бирациональное отображение $\chi: V \rightarrow V^{\prime}$ на некоторое расслоение Мори $V^{\prime} / S^{\prime}$ и линейную систему $\mathscr{D}^{\prime}$ на $V^{\prime}$ того же вида, что и в формулировке предложения 5.7 , и пусть

$$
\mathscr{D}=\chi_{*}^{-1} \mathscr{D}^{\prime} \subset\left|n\left(-K_{V}\right)+m F\right| .
$$

Очевидно, $n>0$. Предположим сначала, что $m \geqslant 0$. Тогда из предложения 5.7 следует, что $\chi$ бирационален по базе, т.е. $V / \mathbb{P}^{1}$ и $V^{\prime} / S^{\prime}$ имеют ту же структуру Мори. Пусть теперь $m<0$. Рассмотрим отображение $\psi^{-1} \circ \chi: U \rightarrow V^{\prime}$ и обозначим $\mathscr{D}_{U}$ собственный прообраз $\mathscr{D}^{\prime}$ на $U$. По формуле (6) находим, что $\mathscr{D}_{U} \subset\left|(n+m)\left(-K_{U}\right)-m F_{U}\right|$. Так как $m<0$, из того же предложения 5.7 получаем, что $U / \mathbb{P}^{1}$ и $V^{\prime} / S^{\prime}$ бирациональны над базой. Таким образом,

$$
\mathscr{M} \mathscr{S}(V)=\left\{\left[V / \mathbb{P}^{1}\right],\left[U / \mathbb{P}^{1}\right]\right\} .
$$

Очевидно, $V$ нерационально (например, потому, что $\mathscr{M} \mathscr{S}(V)$ не содержит структур многообразия Фано), и $V / \mathbb{P}^{1}$ и $U / \mathbb{P}^{1}$ - единственные неособые расслоения Мори в своем классе бирациональной эквивалентности по теореме 4.6.

Пусть теперь $\chi: V \rightarrow V-$ бирациональный автоморфизм. Повторяя только что проведенные рассуждения с заменой $V^{\prime} / S^{\prime}$ на $V / \mathbb{P}^{1}$ и используя теорему 4.6 , 
мы видим, что либо само $\chi$ бирегулярно (это общий случай), либо бирегулярна композиция $\psi^{-1} \circ \chi: U \rightarrow V$. В последнем случае имеем

$$
0 \longrightarrow\langle\psi\rangle_{2} \longrightarrow \operatorname{Bir}(V) \longrightarrow \operatorname{Aut}(V) \longrightarrow 0
$$

где $\langle\psi\rangle_{2}$ - группа, порожденная бирациональной инволюцией $\psi$. Теорема 5.8 доказана.

Для завершения бирациональной классификации неособых расслоений на поверхности дель Пеццо степени 1 осталось рассмотреть самый сложный случай, когда $\varepsilon=0, n_{1}=0, n_{2}=1, n_{3}=2$. Если $V / \mathbb{P}^{1}-$ многообразие с таким набором структурных констант, то $G_{V}$ изоморфно прямому произведению прямой и эллиптической кривой, причем этот дивизор может быть стянут бирациональным морфизмом, задаваемым линейной системой $\left|3\left(-K_{V}\right)-3 F\right|$, на неособое многообразие Фано, называемое двойным конусом над поверхностью Веронезе. Описание бирациональной геометрии таких многообразий удобнее начать со структуры Фано.

Пусть $U$ - неособое трехмерное многообразие Фано индекса 2 и степени 1 (двойной конус над поверхностью Веронезе). Обозначим $H$ класс обильных дивизоров, порождающих группу Пикара, $\operatorname{dim}|H|=2$, так что

$$
\operatorname{Pic}(U)=\mathbb{Z} H
$$

и выполняются соотношения $K_{V}=-2 H$ и $H^{3}=1$. Линейная система $|H|$ имеет единственную базисную точку, которую обозначим $P$.

Легко видеть, что $U$ можно рассматривать как гиперповерхность степени 6 во взвешенном проективном пространстве $\mathbb{P}=\mathbb{P}(1,1,1,2,3)$. Если выбрать $[p, x, y, z, w]$ координатами в $\mathbb{P}$ с весами $\operatorname{wt}(p, x, y, z, w)=(1,1,1,2,3)$ и заметить, что $U$ не проходит через точки $(0,0,0,1,0)$ и $(0,0,0,0,1)$, уравнение для $U$ можно взять в виде

$$
w^{2}+z^{3}+f_{6}(z, x, y, p)=0,
$$

где $f_{6}$ - однородный полином степени 6 , не содержащий мономов $w^{2}$ и $z^{3}$.

Ограничение проекции $\mathbb{P}=\mathbb{P}(1,1,1,2,3) \rightarrow \mathbb{P}(1,1,1,2)$ на $U$ определяет конечный морфизм $U \stackrel{2: 1}{\longrightarrow} \mathbb{P}(1,1,1,2)$ степени 2 , разветвленный над неособым дивизором степени 6 . В свою очередь, $\mathbb{P}(1,1,1,2)$ получается как конус в $\mathbb{P}^{6}$ над поверхностью Веронезе в $\mathbb{P}^{5}$ (т.е. над плоскостью, вложенной в $\mathbb{P}^{5}$ при помощи полной линейной системы коник). Этим объясняется происхождение другого названия $U$ - “двойной конус над поверхностью Веронезе". Дивизор ветвления является неособым сечением конуса над поверхностью Веронезе некоторой кубической гиперповерхностью в $\mathbb{P}^{6}$, не проходящей через вершину конуса. Уравнение дивизора ветвления в $\mathbb{P}(1,1,1,2)$ получается из уравнения для $U$, если отбросить моном $w^{2}$.

На $U$ существует двумерное семейство $\mathscr{P}$ эффективных кривых арифметического рода 1 и степени 1 (относительно пересечения с $H$ ). Класс $l$ таких кривых порождает кольцо одномерных циклов, $A^{2}(U)=\mathbb{Z} l$, причем $H^{2}=l$. Все кривые из $\mathscr{P}$ неприводимы, общая кривая - неособа, и в $\mathscr{P}$ существует одномерное подсемейство рациональных кривых с нодальной или каспидальной двойной точкой. 
Для всякой кривой $C \in \mathscr{P}$ линейная система $|H-C|$ будет пучком поверхностей дель Пеццо степени 1 , причем сама кривая $C$ является полным пересечением любых двух элементов пучка. Таким образом, раздутие $\varphi_{C}: U_{C} \rightarrow U$ кривой $C$ имеет естественную проекцию $\rho_{C}: V=U_{C} \rightarrow \mathbb{P}^{1}$, снабжающую $V$ структурой расслоения Мори. Заметим, что $V$ неособо, если $C$ неособа, $V$ имеет обыкновенную двойную точку, если $C$ - нодальная кривая, и для каспидальной кривой $C$ многообразие $V$ обладает "косой” двойной точкой вида $x y+z^{2}+w^{3}=0$ в подходящих локальных координатах.

Расслоение $V / \mathbb{P}^{1}$ имеет структурные константы $\varepsilon=0, n_{1}=0, n_{2}=1, n_{3}=2$, даже если $V$ имеет особую точку. Просто в особом случае дивизор ветвления $\left.R\right|_{Q}$ имеет соответствующую особенность (дювалевскую точку типа $A_{1}$ или $A_{2}$ ).

Несложно выписать соотношения между дивизорами на $U$ и $V=U_{C}$. Рассмотрим линейную систему $\mathscr{D}_{U} \subset|a H-\mu C|$, где $C \in \mathscr{P}$. Другими словами, $\mathscr{D}_{U}$ состоит из дивизоров класса $a H$, имеющих кратность не ниже $\mu$ вдоль кривой $C$. Рассмотрим собственный прообраз $\mathscr{D}_{V}=\left(\varphi_{C}\right)_{*}^{-1} \mathscr{D}_{U} \subset\left|n\left(-K_{V}\right)+m F\right|$ линейной системы $\mathscr{D}_{U}$ на $V$. Числа $a, \mu, n$ и $m$ связаны соотношениями

$$
\begin{gathered}
n=a-\mu, \\
m=2 \mu-a,
\end{gathered}
$$

т.е. $\mathscr{D}_{V} \subset\left|(a-\mu)\left(-K_{V}\right)+(2 \mu-a) F\right|$.

Главный результат о двойном конусе над поверхностью Веронезе следующий.

Теорема 5.9. Если U - неособое трехмерное многообразие Фано индекса 2 и степени 1, то

$$
\mathscr{M} \mathscr{S}(U)=\{[U]\} \cup\left\{\left[\rho_{C}: U_{C} \rightarrow \mathbb{P}^{1}\right]: C \in \mathscr{P}\right\} .
$$

Расслоения $\rho_{C}: U_{C} \rightarrow \mathbb{P}^{1}$ имент тип $(0,0,1,2)$ (основная серия), в том числе и те, которые содержат двойную точку. Группа бирациональных автоморфизмов $U$ совпадает с группой бирегулярных автоморфизмов, $\operatorname{Bir}(U)=\operatorname{Aut}(U)$, эти группы конечны и в общем случае порождены инволючией двойного накрытия (если U рассматривать как двойное накрытие конуса над поверхностью Веронезе с ветвлением в неособом сечении кубикой). Многообразие U нерачионально и не имеет структур расслоения на коники.

Кроме того, неособые модели $U$ в классе расслоений Мори исчерпываются самим $U$ и теми $U_{C}$, которые получаются раздутиями неособых кривых.

Приводить здесь доказательство этой теоремы невозможно по причине объема, найти его можно в работах [35] и [36]. Отметим, что доказательство результата, анонсированного в [37] и близкого теореме 5.9, содержит грубые ошибки.

\section{6. Расслоения на поверхности дель Пеццо степени 2}

6.1. Конструкция через двойное накрытие. Рассмотрим неособое расслоение $\rho: V \rightarrow \mathbb{P}^{1}$ на поверхности дель Пеццо степени 2. Обозначая через $F$ слой морфизма $\rho$, имеем

$$
\operatorname{Pic}(V)=\mathbb{Z}\left[-K_{V}\right] \oplus \mathbb{Z}[F]
$$


и $\left(-K_{V}\right)^{2} \circ F=2$. Следующая конструкция является расслоенным вариантом представления поверхности дель Пеццо степени 2 как двойного накрытия плоскости, разветвленного над квартикой (предложение 3.1). Найдется такое целое число $m$, что

$$
\rho_{*} \mathscr{O}\left(-K_{V}+m F\right)=\mathscr{E},
$$

где $\mathscr{E} \simeq \mathscr{O} \oplus \mathscr{O}\left(n_{1}\right) \oplus \mathscr{O}\left(n_{2}\right)$ - локально свободный пучок ранга 3 на базе $\mathbb{P}^{1}$, $0 \leqslant n_{1} \leqslant n_{2}$. Положим

$$
X=\operatorname{Proj} \mathscr{E},
$$

и пусть $\pi: X \rightarrow \mathbb{P}^{1}$ - естественная проекция. Обозначим тавтологический дивизор на $X$ через $M$, слой морфизма $\pi$ через $L$, тогда

$$
\operatorname{Pic}(X)=\mathbb{Z}[M] \oplus \mathbb{Z}[L]
$$

Далее, существует двойное накрытие $\varphi: V \rightarrow X$ с ветвлением в некотором неособом дивизоре $R \sim 4 M+2 a L$ такое, что $\rho$ пропускается через $\varphi$, т.е.

$$
\rho=\pi \circ \varphi
$$

Далее, пусть $t_{0}$ - сечение на $X$, соответствующее сюръекции $\mathscr{E} \rightarrow \mathscr{O} \rightarrow 0, l-$ прямая в слое $\pi$, и положим $s_{0}=\frac{1}{2} \varphi^{*}\left(t_{0}\right)$ и $f=\frac{1}{2} \varphi^{*}(l)$. Тогда

$$
\begin{aligned}
& \overline{\mathbf{N E}}(X)=\mathbf{N E}(X)=\mathbb{R}_{+}\left[t_{0}\right] \oplus \mathbb{R}_{+}[l], \\
& \overline{\mathbf{N E}}(V)=\mathbf{N E}(V)=\mathbb{R}_{+}\left[s_{0}\right] \oplus \mathbb{R}_{+}[f] .
\end{aligned}
$$

Обозначим $b=n_{1}+n_{2}$ и $H=\varphi^{*}(M)$; ясно, что $F=\varphi^{*}(L)$. Пусть $G \in\left|M-n_{2} L\right|$ - "минимально закрученная по базе" линейчатая поверхность и $G_{V} \sim H-n_{2} F$ - ее прообраз на $V$. Нетрудно подсчитать, что

ЛЕмма 6.1. На $Х$ выполняются следующие соотношения:

$$
\begin{array}{cl}
M^{3}=b, \quad M^{2} \sim t_{0}+b l, & K_{X} \sim-3 M+(b-2) L, \\
M \circ t_{0}=L \circ l=0, & M \circ l=L \circ t_{0}=1 .
\end{array}
$$

На $V$ выполнено:

$$
\begin{gathered}
H \circ F=2 f, \quad H \circ s_{0}=F \circ f=0, \quad H \circ f=F \circ s_{0}=1, \\
K_{V} \sim-H+(a+b-2) F, \quad H^{2} \sim 2 s_{0}+2 b f, \quad H^{3}=2 b, \\
\left(-K_{V}\right)^{2} \sim 2 s_{0}+(8-4 a-2 b) f, \quad\left(-K_{V}\right)^{3}=12-6 a-4 b .
\end{gathered}
$$

Структурные константы $\left(a, n_{1}, n_{2}\right)$ должны удовлетворять некоторым ограничениям. Верна следующая лемма.

Лемма 6.2. Неособый дивизор $R \sim 4 M+2 a L$ существует тогда и только тогда, когда выполнено одно из следующих условий:

(a) $a \geqslant 0$;

(b) $n_{1}+2 a=0 u n_{1}>0$;

(c) $n_{2}+2 a=0 u n_{2}>4 n_{1}>0$. 
ДокАЗАТЕЛЬСтво. По конструкции, дивизор ветвления $R$ должен быть неособым. Отметим, что если $a \geqslant 0$, то такой дивизор всегда найдется по теореме Бертини, так как линейная система $|4 M+2 a L|$ не имеет базисных точек.

Полагаем $a<0$. Тогда сечение $t_{0}$ лежит на $R$ и обязательно $n_{1}>0$, иначе $G \subset R$. Пусть $\gamma: \widetilde{X} \rightarrow X-$ раздутие $t_{0}, B$ - исключительный дивизор. Поверхность $B$ имеет класс $\mathbb{F}_{n_{2}-n_{1}}$, и пусть $s_{B}$ и $l_{B}$ - ее минимальное сечение и слой. Отметим, что

$$
\operatorname{Pic}(\widetilde{X})=\mathbb{Z}[B] \oplus \mathbb{Z}[\widetilde{G}] \oplus \mathbb{Z}[\widetilde{L}],
$$

где $\widetilde{G}$ и $\widetilde{L}$ - прообразы $G$ и $L$ на $\widetilde{X}$. Очевидно, мы можем рассматривать $\widetilde{X}$ как $\mathbb{P}^{1}$-расслоение $\mu: \widetilde{X} \rightarrow B$, т.е. как проективизацию пучка

$$
\mathscr{E}_{B}=\mathscr{O}_{E} \oplus \mathscr{O}\left(s_{B}+n_{2} l_{B}\right)
$$

причем сечение $B$ соответствует проекции $\mathscr{E}_{B} \rightarrow \mathscr{O}_{B} \rightarrow 0, \widetilde{G}=\mu^{-1}(G)$ и $\widetilde{L} \sim \mu^{*}\left(l_{B}\right)$. Обозначим $B^{\prime}$ сечение морфизма $\mu$, отвечающее проекции $\mathscr{E}_{B} \rightarrow$ $\mathscr{O}_{B}\left(s_{B}+n_{2} l_{B}\right) \rightarrow 0$. Отметим, что $B^{\prime} \cap B=\varnothing$. Для собственного прообраза $\widetilde{R}$ дивизора $R$ на $\widetilde{X}$ имеем

$$
\widetilde{R} \sim \gamma^{*}(R)-B \sim 3 B+4 \widetilde{G}+\left(4 n_{2}+2 a\right) \widetilde{L}
$$

Дивизор $R$ удовлетворяет некоторым ограничениям. Во-первых, так как $G$ покрывается кривыми класса $t_{0}+n_{1} l$, то

$$
R \circ\left(t_{0}+n_{1} l\right)=2 a+4 n_{1}=2\left(a+2 n_{1}\right) \geqslant 0 .
$$

Кроме того, ограничение

$$
\left.\widetilde{R}\right|_{B} \sim s_{B}+\left(n_{2}+2 a\right) l_{B}
$$

должно быть неособой кривой, так как $R$ неособо вдоль $t_{0}$. Тогда выполняется одно из двух условий:

(a) либо $n_{2}+2 a \geqslant n_{2}-n_{1}$, т.е. $n_{1}+2 a \geqslant 0$;

(b) либо $n_{2}+2 a=0$.

При выполнении первого из этих двух условий, т.е. $n_{1}+2 a \geqslant 0$, автоматически выполняется $4 n_{1}+2 a \geqslant 0$. Более того, так как

$$
\widetilde{R} \sim 3 B+4 \widetilde{G}+\left(4 n_{2}+2 a\right) \widetilde{L} \sim 3 B^{\prime}+\mu^{*}\left(s_{B}+\left(n_{2}-n_{1}+n_{1}+2 a\right) l_{B}\right),
$$

а линейная система $\left|3 B^{\prime}+\mu^{*}\left(s_{B}+\left(n_{2}-n_{1}+n_{1}+2 a\right) l_{B}\right)\right|$ не имеет базисных точек при $n_{1}+2 a \geqslant 0$, то по теореме Бертини получаем, что общий дивизор класса $R^{\prime}$ (а значит, и $R$ ) неособый.

Пусть теперь $2 a+n_{2}=0$. Если $a+2 n_{1}=0$, то ограничение $\left.R\right|_{G}=4 t_{0}$, а тогда дивизор $G_{V}$ на расслоении $V / \mathbb{P}^{1}$ распадется на две линейчатые поверхности, что противоречит условию на расслоение Мори, так как тогда относительное число Пикара $V$ над $\mathbb{P}^{1}$ будет больше единицы.

Таким образом, $2 a+n_{2}=0$ и $a+2 n_{1}>0$. Мы можем представить дивизор $\widetilde{R}$ двумя разными способами:

$$
\widetilde{R} \sim 3 B^{\prime}+\widetilde{G} \sim 3 B+\mu^{*}\left(4 s_{B}+\left(4 n_{2}-4 n_{1}+4 n_{1}+2 a\right) l_{B}\right),
$$


откуда видно, что линейная система $\widetilde{R}$ не имеет базисных компонент, а общий ее элемент неособ вдоль кривой $s_{B}=\operatorname{Bas}\left|R^{\prime}\right|$. Таким образом, теорема Бертини и в этом случае влечет, что общий элемент из $|R|$ неособ. Лемма 6.2 доказана.

В последующем нам будет удобно описывать результаты о расслоениях на поверхности дель Пеццо степени 2 в зависимости от величины параметра $2 a+b$, вычисляющего, как нетрудно видеть из леммы 6.1 , сдвинутое $K^{2}$-условие.

СлЕДСТвиЕ 6.3. (а) При $2 a+b=2$ реализуются толъко следующие случаи:

1) $a=-1, n_{1}=n_{2}=2$;

2) $a=1, n_{1}=n_{2}=0$;

3) $a=0, n_{1}=n_{2}=1$;

4) $a=0, n_{1}=0, n_{2}=2$;

5) $a=-2, n_{1}=2, n_{2}=4$;

6) $a=-3, n_{1}=2, n_{2}=6$.

(b) При $2 a+b=1$ реализуются только два случая:

1) $a=0, n_{1}=0, n_{2}=1$;

2) $a=-1, n_{1}=1, n_{2}=2$.

(с) Случаи $2 a+b \leqslant 0$ не реализуются.

ДокАЗАТЕЛЬСтво. При $2 a+b>0$ все следует из предыдущей леммы. Пусть $2 a+b \leqslant 0$. Если $b=a=0$, то $V$ изоморфно прямому произведению $\mathbb{P}^{1}$ и гладкой поверхности дель Пеццо степени 2. Такое многообразие не является, очевидно, расслоением Мори, поскольку ранг относительной группы Пикара равен 8. Случай $b=0$ и $a<0$ невозможен, так как $R$ не будет эффективным дивизором. Если же $b>0$ при $2 a+b=0$, то $a<0$, и все опять следует из леммы выше. Следствие 6.3 доказано.

6.2. Вложение в $\mathbb{P}(1,1,1,2)$-расслоение. Согласно предложению 3.1 , неособые (на самом деле, горенштейновы) расслоения на поверхности дель Пеццо степени 2 вкладываются как гиперповерхность в $\mathbb{P}(1,1,1,2)$-расслоение над базой (в нашем случае $\left.\mathbb{P}^{1}\right)$. Конструкция ниже взята из [38].

Пусть $\mathscr{S}=\bigoplus_{n \geqslant 0} \mathscr{S}_{n}$ - пучок градуированных алгебр на $\mathbb{P}^{1}$ со свойствами:

(a) $\mathscr{S}_{1}=\mathscr{E}=\mathscr{O} \oplus \mathscr{O}\left(n_{1}\right) \oplus \mathscr{O}\left(n_{2}\right)$, где $0 \leqslant n_{1} \leqslant n_{2}$;

(b) $\mathscr{S}_{2}=S^{2} \mathscr{E} \oplus \mathscr{F}$, где $\mathscr{F}=\mathscr{O}(-a)$;

(c) $\mathscr{S}$ порождена $\mathscr{E}$ и $\mathscr{F}$.

Рассмотрим расслоение $\pi: X=\operatorname{Proj} \mathscr{S} \rightarrow \mathbb{P}^{1}$ на взвешенные проективные пространства с весами $(1,1,1,2)$; пусть $H$ - тавтологический $\mathbb{Q}$-Картье дивизор, другими словами, $2 H$ - дивизор Картье, $\mathscr{O}_{X}(H)$ - рефлексивный пучок ранга 1 такой, что $\pi_{*} \mathscr{O}_{X}(H)=\mathscr{S}_{1}$ и $\pi_{*} \mathscr{O}_{X}(2 H)=\mathscr{S}_{2}$, и пусть $F-$ слой $X$. Мы полагаем, что наше неособое расслоение $V \rightarrow \mathbb{P}^{1}$ на поверхности дель Пеццо степени 1 является элементом линейной системы $V \in|4 H+k F|$ для некоторого целого $k$.

Обозначим $s$ сечение $V / \mathbb{P}^{1}$, соответствующее проекции $\mathscr{S}_{2} \rightarrow \mathscr{F} \rightarrow 0$. Многообразие $V$ имеет особенность типа $\frac{1}{2}(1,1,1)$ вдоль $s$. Далее, линейчатая поверхность $T$, отвечающая проекции $\mathscr{S} \rightarrow S(\mathscr{O} \oplus \mathscr{F})$, имеет тип $\mathbb{F}_{|a|}$. Обозначив $s_{T}$ и $f_{T}$ ее сечение, отличное от $s$, и слой, мы находим, что

$$
\left.V\right|_{T} \sim 2 s_{T}+(k-2 a) f_{T}
$$


Отметим, что $s \cap V=\varnothing$, поэтому $T \not \subset V$, и кривая $\left.V\right|_{T}$ эффективна и не пересекает сечение $s$. Отсюда $k=2 a$. Мы получили, что $V \sim 4 H+2 a F$, параметры $\left(a, n_{1}, n_{2}\right)$ в точности соответствуют параметрам из конструкции через двойное накрытие. В этом легко убедиться, вычисляя прямые образы для $\mathscr{O}_{V}(H)$ и $\mathscr{O}_{V}(2 H)$.

6.3. Результаты о жесткости. Пусть $\rho: V \rightarrow \mathbb{P}^{1}$ - неособое расслоение на поверхности дель Пеццо степени 2 с набором структурных констант $\left(a, n_{1}, n_{2}\right)$, и пусть $b=n_{1}+n_{2}$. Отметим, что $K^{2}$-условие соответствует неравенству $2 a+b \geqslant 4$. Что же касается $K$-условия, верна следующая теорема.

Теорема 6.4 [6], [29], [39]. Гипотеза 4.4 верна для неособых расслоений на поверхности дель Пеццо степени 2 , с учетом предположений общности $\mathscr{C}_{1}$, $\mathscr{C}_{2}$ и $\mathscr{C}_{3}$ (сформулированы ниже) для соответствующих многообразий типов $(-3,2,6),(-2,2,4)$ и $(0,0,2)$ соответственно. Именно, помимо многообразий, удовлетворяющих $K^{2}$-условию, бирационально жесткими будут все многообразия при $2 a+b=3$, а также многообразия, соответствующие наборам $\left(a, n_{1}, n_{2}\right)$ при $2 a+b=2$ следующих видов: $(-3,2,6),(-2,2,4)$ и $(0,0,2)$ (эти три при выполнении упомянутых условий общности). Бирационально нежесткими будут все многообразия с $2 a+b=1$ (два возможных набора структурных констант, согласно следствию 6.3), а при $2 a+b=2$ следующие: $(-1,2,2),(1,0,0),(0,1,1)$.

Условия, перечисленные в теореме, возникли ввиду технических трудностей доказательства результата и, по мнению автора, не имеют принципиального характера. По мере развития техники метода максимальных особенностей их, вероятно, удастся снять. Условия эти такие.

Условие $\mathscr{C}_{1}$ для расслоений типа $(-2,2,6)$ заключается в следующем: дивизор ветвления $R$ высекает на $G$ (а это линейчатая поверхность типа $\mathbb{F}_{2}$ ) тройное исключительное сечение и сечение, не пересекающее исключительное.

Условие $\mathscr{C}_{2}$ для типа $(-2,2,4)$ аналогично предыдущему: $R$ высекает на $G$ $\left(\right.$ как и выше, это $\mathbb{F}_{2}$ ) двойное исключительное сечение и бисечение (возможно, приводимое), не пересекающее исключительное.

Условие $\mathscr{C}_{3}$ для типа $(0,0,2)$ содержит два требования. Во-первых, $V$ имеет общие вырождения, т.е. не более одной особой точки типа $\mathbb{A}_{1}$ в слое, или, что то же самое, $R$ имеет простейшие вырождения. Далее, нетрудно видеть, что $G \cong \mathbb{P}^{1} \times \mathbb{P}^{1}$ заметается кривыми класса $t_{0}$, причем $R$ высекает на $G$ в точности 4 различные сечения этого класса (последнее следует из неособости $R$ и $G$ : достаточно раздуть кривую их пересечения и убедиться, что собственные прообразы высекают на исключительном дивизоре непересекающиеся сечения). Любой слой $X$ (а это плоскости) указанные четыре сечения пересекают в 4 различных точках. Второе требование состоит в том, что через эти точки не проходят четырехкратные касательные к дивизору ветвления (но если одна из четырех точек совпадает с особой точкой ограничения дивизора ветвления на слой, то требование на такую точку снимается). На $V$ это требование выглядит следующим образом: те антиканонические кривые в слоях, которые распадаются на пару касающихся друг друга прямых, не пересекают ни одно из четырех сечений класса $s_{0}$, что лежат над указанными сечениями класса $t_{0}$ 
(кроме случая, когда особая точка такой антиканонической кривой совпадает с особой точкой слоя).

Можно показать, что общие расслоения указанных типов соответствуют этим условиям [39].

Отметим, что любое расслоение на поверхности дель Пеццо степени 2 обладает значительной группой бирациональных автоморфизмов. С каждым сечением расслоения связана инволюция, которая в слое над общей точкой индуцирует инволюцию Бертини, как в предложении 3.3 .

НАБРОСОК ДОКАЗАТЕЛЬСТВА ТЕОРЕМЫ 6.4. Собственно, доказательство состоит из двух частей. Надо доказать бирациональную жесткость расслоений, удовлетворяющих $K$-условию, и предъявить разные структуры Мори для остальных. По причине объема доказательство жесткости здесь не приводится. При выполнении $K^{2}$-условия, т.е. когда $2 a+b \geqslant 4$, это сделано в работе [6], при $2 a+b=3$ в [29] (собственно, рассуждения из [6] проходят и в этом случае), при $2 a+b=2$ в [39].

Проверим, что в жестких случаях выполнено $K$-условие. Как уже отмечалось, это всегда так, если есть $K^{2}$-условие. Пусть $2 a+b=3$, и рассмотрим линейную систему $\mathscr{D}=\left|n\left(-K_{V}\right)+m F\right|$ при $n>0$. Так как $2 a+b=3$, то $b$ всегда нечетно, так что обязательно $n_{1}<n_{2}$. Предположим, что $m<0$, и рассмотрим общую кривую $C \sim 2 s_{0}+2 n_{1} f$ на $V$ (таких, как минимум, одномерное семейство). Тогда для общего $D \in \mathscr{D}$ всегда выполнено $D \circ C \geqslant 0$, и, подставляя $a=\frac{1}{2}(3-b)$, имеем

$$
D \circ C=\left(n H-\left(\frac{n}{2}(b-1)-m\right) F\right) \circ\left(s_{0}+n_{1} f\right) \geqslant 0,
$$

откуда приходим к противоречию:

$$
0<\frac{-m}{n} \leqslant n_{1}-\frac{b}{2}+\frac{1}{2}=\frac{n_{1}-n_{2}+1}{2} \leqslant 0 .
$$

Таким образом, при $2 a+b=3$ всегда выполнено $K$-условие. Оставшиеся жесткие случаи относятся к ситуации $2 a+b=2$.

Пусть сначала $\left(a, n_{1}, n_{2}\right)=(0,0,2)$. Дивизор $G_{V} \sim\left(-K_{V}\right)-2 F$ изоморфен прямому произведению прямой и эллиптической кривой и покрывается одномерным семейством класса $s_{0}$. Поскольку $\left(-K_{V}\right) \circ s_{0}=0$, линейные системы вида $\left|n\left(-K_{V}\right)-F\right|$ содержат $G_{V}$ в качестве неподвижной компоненты. Положим теперь $\left(a, n_{1}, n_{2}\right)=(-3,2,6)$. На дивизоре $G_{V} \sim\left(-K_{V}\right)-3 F$ линейная система $|H|$ высекает кривые класса $2 s_{0}+4 f$. Так как $\left(-K_{V}\right) \circ\left(2 s_{0}+4 f\right)=-2$, линейные системы вида $\left|n\left(-K_{V}\right)-F\right|$ содержат базисную компоненту $G_{V}$. Аналогично для последнего оставшегося случая $\left(a, n_{1}, n_{2}\right)=(-3,2,6)$. На $G_{V} \sim\left(-K_{V}\right)-2 F$ линейная система $|H|$ высекает кривые класса $2 s_{0}+2 f$. Имеем $\left(-K_{V}\right) \circ\left(2 s_{0}+2 f\right)=0$, так что линейные системы $\left|n\left(-K_{V}\right)-F\right|$ все содержат $G_{V}$.

Остается показать, что другие случаи структурных констант относятся к нежестким многообразиям, и проверить утверждение гипотезы 4.4 для них. Все они относятся к ситуации $0<2 a+b \leqslant 2$.

Случай $\left(a, n_{1}, n_{2}\right)=(1,0,0)$. Здесь $X \cong \mathbb{P}^{2} \times \mathbb{P}^{1}$ и $V$ является двойным накрытием $X$ с ветвлением в дивизоре бистепени $(4,2)$. Относительно проекции 
на $\mathbb{P}^{2}$ многообразие $V$ есть расслоение на коники, слои которого дважды накрывают на $X$ кривые класса $t_{0}$ с ветвлением в $R \circ t_{0}=2$ точках. Заметим, что эта проекция дается линейной системой $\left|-K_{V}-F\right|$, не имеющей базисных точек. Таким образом, $V$ бирационально нежестко и удовлетворяет гипотезе 4.4 . Про это многообразие можно высказать следующее предположение:

ГиПотезА 1. Неособое расслоение на поверхности дель Пещцо степени 2 со структурными константами $\left(a, n_{1}, n_{2}\right)=(1,0,0)$ имеет ровно эти две описанные структуры Мори.

Случай $\left(a, n_{1}, n_{2}\right)=(-1,2,2)$. В этом случае единственное сечение класса $t_{0}$ на $X$ обязательно лежит на дивизоре ветвления $R \sim M-2 L$, поэтому сечение класса $s_{0}$ на $V$ тоже одно. Заметим, что $\mathscr{N}_{s_{0} \mid V}=\mathscr{O}(-1) \oplus \mathscr{O}(-2)$ и линейная система $|n H|$ при $n \geqslant 2$ дает бирациональный морфизм, стягивающий лишь $s_{0}$. Далее, на $X$ базисное множество пучка $|M-L|$ есть в точности $t_{0}$, все элементы этого пучка гладкие и изоморфны поверхности $\mathbb{F}_{2}$. Если $S \in|M-2 L|-$ общий элемент, то $\left.R\right|_{S}$ распадается на $t_{0}$ и некоторое три-сечение, не пересекающее $t_{0}$, так что после двойного накрытия и последующего стягивания прообраза $s_{0}$ прообраз поверхности $S$ станет поверхностью дель Пеццо степени 1 . Это значит, что антифлип $V / \mathbb{P}^{1} \rightarrow V^{\prime} / \mathbb{P}^{1}$ с центром в $s_{0}$ даст нам расслоение на поверхности дель Пеццо степени 1 с одной терминальной точкой, лежащей на исключительной кривой на $V^{\prime}$. Таким образом, $V$ нежестко. Заметим, что прообразы слоев расслоения $V^{\prime} / \mathbb{P}^{1}$ образуют на $V$ пучок $\left|-K_{V}-F\right|$ без неподвижных компонент.

ГиПотезА 2. Неособое расслоение на поверхности дель Пецио степени 2 со структурными константами $\left(a, n_{1}, n_{2}\right)=(-1,2,2)$ имеет ровно эти две указанные структуры Мори: расслоение на поверхности дель Пеццо степени 2 и расслоение на поверхности дель Пеццо степени 1 с простейшей бактор-особенностью индекса 2.

Случай $\left(a, n_{1}, n_{2}\right)=(0,1,1)$. Линейная система $\left|-2 K_{V}\right|$ дает малое стягивание на антиканоническую модель $V$, реализуемую как двойное накрытие невырожденного квадратичного конуса в $\mathbb{P}^{4}$ с ветвлением в сечении квартикой, которая либо проходит, либо не проходит через вершину конуса в зависимости от того, лежит или нет единственная на $X$ кривая класса $t_{0}$ на дивизоре ветвления. В первом случае кривая класса $s_{0}$ на $V$ (а она одна) есть $(-2)$-кривая ширины 2 в терминах работы [40], во втором случае обе кривые класса $s_{0}$ дизъюнктны и являются (-2)-кривыми ширины 1 . И там, и там после соответствующего флопа мы получаем еще одну структуру расслоения на поверхности дель Пеццо степени 2, причем собственные прообразы слоев этой второй структуры на $V$ образуют пучок $\left|-K_{V}-F\right|$ без неподвижных компонент. Случай с двумя кривыми подробно исследован в работе [41]. Там показано, что общее многообразие указанного типа имеет только две указанные структуры Мори.

Случай $\left(a, n_{1}, n_{2}\right)=(-1,1,2)$. Заметим, что кривая класса $s_{0}$ одна на $V$ и является (-2)-кривой ширины 1 . Пусть $V \rightarrow-V^{+}-$флоп с центром в этой кривой. Далее, линейная система $\left|\left(-K_{V}\right)-2 F\right|$ содержит единственный элемент - поверхность $G_{V}$. Ее собственный образ $G_{V}^{+}$на $V^{+}$есть поверхность, изоморфная двойному накрытию $\mathbb{P}^{2}$ с ветвлением либо в неособой конике, либо в 
паре различных прямых, т.е. $G_{V}^{+}$изоморфна либо $\mathbb{P}^{1} \times \mathbb{P}^{1}$, либо квадратичному конусу в $\mathbb{P}^{3}$. Несложно видеть, что существует экстремальное стягивание $V^{+} \rightarrow U$ дивизора $G_{V}^{+}$. Многообразие $U$ есть многообразие Фано, имеет квадратичную особенность, и его явная геометрическая конструкция очень похожа на конструкцию многообразия Фано индекса 2 степени 1: $U$ получается двойным накрытием конуса над поверхностью Веронезе, разветвленным в сечении кубикой, не проходящем через вершину конуса и имеющем одну двойную точку. Пусть $P$ обозначает образующую группы Пикара на $U$, а $p$ - прообраз образующей конуса (это неприводимая кривая арифметического рода 1). Тогда собственный образ линейной системы $|F|$ при отображении $V \rightarrow-U$ есть $|P-p|$, причем в этом случае кривая $p$ проходит через особую точку $U$. Заметим, что раздутие общей кривой класса $p$ (т.е. не проходящей через особую точку $U$ ) дает нам расслоение на поверхности дель Пеццо степени 1 с двойной точкой и теми же структурными константами, что и в случае неособого двойного конуса над поверхностью Веронезе. Отметим также, что собственный прообраз на $V$ линейной системы $|P|$ есть линейная система $\left|-K_{V}-F\right|$, не имеющая базисных компонент.

Случай $\left(a, n_{1}, n_{2}\right)=(0,0,1)$. В этом случае поверхность $G_{V} \sim\left(-K_{V}\right)-2 F$ изоморфна прямому произведению эллиптической кривой и прямой, а линейная система $\left|2\left(-K_{V}\right)-2 F\right|$ дает бирациональный морфизм $V \rightarrow U$, стягивающий $G_{V}$, на неособое многообразие Фано индекса 2 и степени 2 , часто называемое двойным пространством индекса 2. Таким образом, $V$ бирационально нежестко и удовлетворяет гипотезе 4.4. На самом деле, $U$ (значит, и $V$ ) имеет много различных структур Мори. Кроме “четырехмерного семейства" структур расслоения на поверхности дель Пеццо степени 2, получаемых раздутием неприводимых кривых степени 2 и рода 1 , двойное пространство индекса 2 имеет:

(а) "трехмерное семейство" структур расслоения на кубические поверхности, получаемые раздутием прямых на $V$ с последующим флопом в сопряженных (относительно автоморфизма двойного накрытия) прямых;

(b) "двумерное семейство" многообразий Фано с $c D_{4}$-точкой, являющихся полным пересечением двух кубических гиперповерхностей в $\mathbb{P}\left(1^{5}, 2\right)$ [42];

(c) “одномерное семейство” структур расслоения на поверхности дель Пеццо степени 2 со слоем кратности 2 [42].

На самом деле не ясно, нет ли еще каких иных структур Мори на таком многообразии Фано. Однако известно, что общие двойные пространства индекса 2 не имеют структур расслоения на коники. Также интересно, что такие многообразия бирационально изоморфны квартике с плоскостью и $c D_{4}$-точкой вне плоскости. Этим завершим разбор теоремы 6.4 .

\section{7. Расслоения на кубические поверхности}

Пусть $\rho: V \rightarrow \mathbb{P}^{1}$ неособое расслоение на кубические поверхности. Так как слои $V / \mathbb{P}^{1}$ являются горенштейновыми поверхностями дель Пеццо степени 3 , каждый из них вкладывается в $\mathbb{P}^{3}$ при помощи антиканонической линейной системы (предложение 3.1). Для некоторого целого числа $m$ выполняется

$$
\mathscr{E}=\rho_{*} \mathscr{O}_{V}\left(-K_{V}+m F\right)=\mathscr{O} \oplus \mathscr{O}\left(n_{1}\right) \oplus \mathscr{O}\left(n_{2}\right) \oplus \mathscr{O}\left(n_{3}\right),
$$


где $\mathscr{E}$ - расслоение ранга 4 на $\mathbb{P}^{1}$ и $0 \leqslant n_{1} \leqslant n_{2} \leqslant n_{3}$. Положим $X=$ Proj $\mathscr{E}$, тогда $V$ можно рассматривать как гиперповерхность в расслоении $\pi: X \rightarrow \mathbb{P}^{1}$. Пусть $M$ и $L$ - тавтологический дивизор и слой $X / \mathbb{P}^{1}, t_{0}$ и $l$ - соответственно минимальное сечение (отвечающее проекции $\mathscr{E} \rightarrow \mathscr{O} \rightarrow 0$ ) и прямая в слое. Тогда

$$
V \sim 3 M+k L
$$

для некоторого целого $k$. Обозначим $b=n_{1}+n_{2}+n_{3}$.

Очевидно, набор структурных констант $\left(k, n_{1}, n_{2}, n_{3}\right)$ с точностью до деформаций определяет расслоение $V / \mathbb{P}^{1}$. При этом, разумеется, существуют ограничения на число $k$, которое не может быть слишком отрицательным. Например, всегда $-k \leqslant n_{3}$ и $-k \leqslant 3 n_{1}$. Действительно, если $k<0$, то $t_{0} \subset V$, и при условии $-k>n_{3}$ мы получаем, что $V$ обязательно имеет особенность вдоль $t_{0}$, в чем легко убедиться, раздув $t_{0} \subset X$ и ограничив собственные прообразы $V \in|3 M+k L|$ и $\left|M-n_{3} L\right|$ на исключительный дивизор. Если же $-k>3 n_{1}$, то $V$ содержит поверхность $G=\operatorname{Proj}\left(\mathscr{O} \oplus \mathscr{O}\left(n_{1}\right)\right)$, что противоречит условию на относительное число Пикара, так как слой $V_{\eta}$ над общей схемной точкой $\eta$ прямой будет содержать $(-1)$-прямую $G_{\eta}$, т.е. $\operatorname{rk} \operatorname{Pic}\left(V_{\eta}\right)>1$.

В целом, геометрия неособых расслоений на кубические поверхности заметно сложнее, чем расслоений на поверхности дель Пеццо степеней 1 и 2. Рассмотрим, например, $K^{2}$-условие. Легко подсчитать, что

$$
K_{V}^{2} \sim 3 t_{0}+(12-5 k-3 b) l,
$$

и если это условие не выполнено, т.е. $K_{V}^{2} \in \operatorname{Int} \mathbf{N E}(V)$, то выполнено неравенство $5 k+3 b<12$. Обратное, вообще говоря, неверно. Точнее, если $5 k+3 b<12$ и $k<0$, то $t_{0} \subset V$ и $K^{2}$-условие не выполнено, но если $k \geqslant 0$, то может быть так, что $t_{0} \not \subset V$, и тогда легко построить контрпримеры. Более того, если в случаях расслоений на поверхности дель Пеццо степени 1 или 2 многообразия с невыполненным $K^{2}$ - или $K$-условием лежали в конечном числе семейств (т.е. соответствовали конечному числу структурных наборов), то для расслоений на кубические поверхности это уже не так. Подробнее об этом можно прочитать в работе [43].

Что же касается $K$-условия, то и здесь все непросто. Например, для структурного набора $(1,0,0,1)$ можно показать, что $\left|3\left(-K_{V}\right)-F\right|$ не пуста и не имеет неподвижных компонент (не выполнено $K$-условие), но линейные системы $\left|\left(-K_{V}\right)-F\right|$ и $\left|2\left(-K_{V}\right)-F\right|$ имеют неподвижную компоненту класса $\left(-K_{V}\right)-F$. Ничего подобного на неособых расслоениях на поверхности дель Пеццо степени 1 или 2 не может быть ("виновата" в этом конструкция двойного накрытия).

Относительно структур Мори на расслоениях на кубические поверхности основным на данный момент является следующий результат А. В. Пухликова.

Теорема 7.1 [6]. Пусть $V / \mathbb{P}^{1}$ - неособое расслоение на кубические поверхности, общее в том смысле, ито слой может иметь не более одной обыкновенной двойной точки и через такую точку проходит ровно 6 прямых. Если для $V / \mathbb{P}^{1}$ выполнено $K^{2}$-условие, то оно бирационально жестко.

Условия общности в этой теореме, как и в теореме 6.4, возникли по чисто техническим обстоятельствам и, по всей видимости, могут быть сняты в дальнейшем. 
Кроме указанных, существуют расслоения на кубические поверхности, для которых получены достаточно полные результаты. Именно, рассмотрим структурный набор $(m, 0,0,0)$. Другими словами, $V / \mathbb{P}^{1}$ является дивизором бистепени $(m, 3)$ в прямом произведении $\mathbb{P}^{1} \times \mathbb{P}^{3}$. Тогда если $m=1$, многообразие $V$ рационально. Действительно, в этом случае ограничение проекции $\mathbb{P}^{1} \times \mathbb{P}^{3} \rightarrow \mathbb{P}^{3}$ на $V$ дает бирациональное отображение $V \rightarrow-\mathbb{P}^{3}$. Если $m \geqslant 3$, то для $V / \mathbb{P}^{1}$ выполняется $K^{2}$-условие, так что общее многообразие такого типа бирационально жестко по теореме выше. Пусть $m=2$. Заметим, что по результатам работы [4] общее $V_{m}$ нерационально при $m \geqslant 2$. Нетрудно убедиться, что $V=V_{2}$ бирационально нежестко. Общее такое многообразие содержит ровно 27 сечений, являющихся слоями проекции $\mathbb{P}^{1} \times \mathbb{P}^{3} \rightarrow \mathbb{P}^{3}$. С другой стороны, общий слой такой проекции пересекает $V$ в двух точках, что определяет бирациональную инволюцию $\tau \in \operatorname{Bir}(V)$. Эта инволюция реализуется флопом в указанных 27 прямых. При этом получается другая структура $U / \mathbb{P}^{1}$ расслоения на кубические поверхности, причем $V \cong U$, а $\tau$ есть линк Саркисова типа IV. Отметим, что линейная система $\left|3\left(-K_{V}\right)-F\right|$ является пучком без базисных компонент, т.е. $K$-условие не выполняется. Элементы этого пучка инволюция $\tau$ переводит в слои $U / \mathbb{P}^{1}$. Доказано [44], что $\mathscr{M} \mathscr{S}(V)$ состоит только из расслоений на кубические поверхности, которые как многообразия изоморфны $V$, так что бирациональные отображения между ними являются, очевидно, элементами группы $\operatorname{Bir}(V)$. Эта группа, в свою очередь, представляется как расширение

$$
0 \longrightarrow \tau \times \operatorname{Bir}\left(V_{\eta}\right) \longrightarrow \operatorname{Bir}(V) \longrightarrow \operatorname{Aut}(V) \longrightarrow 0
$$

где $\operatorname{Bir}\left(V_{\eta}\right)$ группа бирациональных автоморфизмов слоя над общей схемной точкой, порожденная инволюциями Бертини и Гейзера (предложение 3.3).

Таким образом, многообразие $V=V_{2}$ нерационально, бирационально нежестко, и любой пучок рациональных поверхностей на нем при помощи бирационального автоморфизма перекручивается в слои $V / \mathbb{P}^{1}$.

Недавно И. А. Чельцовым был доказан очень интересный результат о нерациональности очень общих неособых расслоений на кубические поверхности. В данном случае "очень общее" расслоение означает, что оно взято из дополнения по Зарисскому к счетному объединению замкнутых подмножеств в пространстве модулей, параметризующем расслоения на кубические поверхности.

Теорема 7.2 [45]. Пусть $V / \mathbb{P}^{1}$ - очень общее неособое расслоение на кубические поверхности. Многообразие $V$ нерационально тогда и только тогда, когда его структурный набор отличен от $(1,0,0,0)$.

НАБРОСОК ДОКАЗАТЕЛЬСТВА. Введем биоднородные координаты $\left(t_{0} ; t_{1}\right.$; $\left.x_{0}: x_{1}: x_{2}: x_{3}\right)$ на расслоении $\pi: X=\operatorname{Proj} \mathscr{E} \rightarrow \mathbb{P}^{1}$, где $\left(t_{0}: t_{1}\right)$ - координаты в $\mathbb{P}^{1}, \quad\left\{x_{0}=0\right\}-$ множество нулей многообразия $\operatorname{Proj}\left(\mathscr{O}\left(n_{1}\right) \oplus \mathscr{O}\left(n_{2}\right) \oplus\right.$ $\left.\mathscr{O}\left(n_{3}\right)\right) \subset X,\left\{x_{1}=0\right\}-$ множество нулей $\operatorname{Proj}\left(\mathscr{O} \oplus \mathscr{O}\left(n_{2}\right) \oplus \mathscr{O}\left(n_{3}\right)\right) \subset X$ и т. д. Тогда $V$ можно представить как множество нулей биоднородного многочлена

$$
\sum_{i=0}^{3} \alpha_{i}\left(t_{0}, t_{1}\right) x_{0}^{i} x_{1}^{3-i}+x_{3} F+x_{4} G=0
$$


где $\alpha_{i}$ - однородный многочлен степени $k+(3-i) n_{1}$, а $F$ и $G$ обозначают многочлены

$$
\sum_{\substack{i, j, l, m \geqslant 0 \\ i+j+l+m=2}} \beta_{i j l m}\left(t_{0}, t_{1}\right) x_{0}^{i} x_{1}^{j} x_{2}^{l} x_{3}^{m} \quad \text { и } \sum_{\substack{i, j, l, m \geqslant 0 \\ i+j+l+m=2}} \gamma_{i j l m}\left(t_{0}, t_{1}\right) x_{0}^{i} x_{1}^{j} x_{2}^{l} x_{3}^{m}
$$

с коэффициентами $\beta_{i j l m}$ и $\gamma_{i j l m}$, являющимися однородными многочленами степеней $k+j n_{1}+(l+1) n_{2}+m n_{3}$ и $k+j n_{1}+l n_{2}+(m+1) n_{3}$ соответственно [46].

Рассмотрим расслоение на кубики $U \subset X$, заданное уравнением

$$
x_{3} F+x_{4} G=0 .
$$

Покажем, что $U$ нерационально и нелинейчато (т.е. не бирационально многообразию $\mathbb{P}^{1} \times B$, где $B$ - некоторая поверхность), кроме одного случая. Ввиду общности $V$ можно полагать, что $U$ неприводимо и неособо всюду, кроме точек $x_{3}=x_{4}=F=G=0$, являющихся обыкновенными двойными и лежащих на линейчатой поверхности $G=\left\{x_{3}=x_{4}=0\right\}=\operatorname{Proj}\left(\mathscr{O} \oplus \mathscr{O}\left(n_{1}\right)\right) \subset X$. Можно подсчитать, что таких двойных точек на $U$ будет ровно $4 k+4 n_{1}+2 n_{2}+2 n_{3}$.

Слой $U_{\eta}$ над общей схемной точкой $\eta$ прямой содержит $(-1)$-прямую $G_{\eta}$, так что $\mathrm{rk} \operatorname{Pic}\left(U_{\eta}\right)=2$, а дивизор $G \subset U$ не является $\mathbb{Q}$-Картье дивизором. Пусть $\varphi: X^{\prime} \rightarrow X-$ раздутие поверхности $G$, тогда ограничение на $U$ определяет малое разрешение $\varphi_{U}: U^{\prime} \rightarrow U$ особенностей $U$, причем можно показать, что $\operatorname{rk} \operatorname{Pic}\left(U^{\prime}\right)=3$. Тогда, очевидно, $U^{\prime}$ является расслоением на коники

$$
\tau: U^{\prime} \rightarrow S
$$

над линейчатой поверхностью $S=\operatorname{Proj}\left(\mathscr{O}\left(n_{3}\right) \oplus \mathscr{O}\left(n_{4}\right)\right)$ класса $\mathbb{F}_{n_{4}-n_{3}}$ с кривой вырождения $\delta \subset S$. Пусть $s$ и $f$ - минимальное сечение и слой $S$. Несложно вычислить, что

$$
2 K_{S}+\delta \sim s+\left(3 k+3 n_{3}+6 n_{2}-2 n_{1}-4\right) f
$$

так что

$$
\left|2 K_{S}+\delta\right| \neq \varnothing \Longleftrightarrow 3 k+3 n_{3}+6 n_{2}-2 n_{1} \geqslant 4 .
$$

Известно, что неособое расслоение на коники с относительным числом Пикара, равным 1 , над линейчатой поверхностью или плоскостью нерационально, если $\left|2 K_{S}+\delta\right| \neq \varnothing[47]$. Из неособости и относительной минимальности $V$ следует, что условие

$$
3 k+3 n_{3}+6 n_{2}-2 n_{1}<4
$$

выполняется в двух случаях структурных констант $\left(k, n_{1}, n_{2}, n_{3}\right):(1,0,0,0)$ и $(0,0,0,1)$. В первом случае многообразие $V$ рационально, являясь гиперповерхностью бистепени $(1,3)$ в $\mathbb{P}^{1} \times \mathbb{P}^{3}$. Набор $(0,0,0,1)$ соответствует расслоению на кубические поверхности, получаемому раздутием неособого плоского сечения неособой трехмерной кубической гиперповерхности, которая, как известно, нерациональна.

Таким образом, во всех случаях структурных констант, отличных от $(1,0$, $0,0)$, расслоение на коники $U^{\prime} / S$, а значит, и расслоение на кубические поверхности $U / \mathbb{P}^{1}$ нерационально. Так как $U$ рационально связно и нерационально, то оно нелинейчато.

Нам остается воспользоваться следующим известным фактом. 
ПРЕДЛОЖЕНИЕ 7.3 [48]. Пусть $\zeta: Y \rightarrow Z$ - морфизм с неприводимыми приведенными равноразмерными слоями. Тогда существует счетное множество замкнутых по Зарисскому подмногообразий $Z_{i} \subset Z$ таких, что для точки $z \in Z$ слой $\zeta^{-1}(z)$ является линейчатым, если и только если $z \in \bigcup Z_{i}$.

Так как, очевидно, $V$ представляется плоской деформацией $U$ и $U$ нелинейчато, то очень общее $V$ будет также нелинейчато, а поэтому нерационально.

\section{Список литературы}

[1] Ю.И. Манин, "Рациональные поверхности над совершенными полями", Inst. Hautes Études Sci. Publ. Math., 30 (1966), 55-97.

[2] Ю.И. Манин, "Рациональные поверхности над совершенными полями. II", Матем. сб., 72:2 (1967), 161-192.

[3] В. А. Исковских, "Минимальные модели рациональных поверхностей над произвольными полями", Изв. АН СССР. Сер. матем., 43:1 (1979), 19-43.

[4] F. Bardelli, "Polarized mixed Hodge structure: on irrationality of threefolds via degeneration", Ann. Mat. Pura Appl. (4), 137 (1984), 287-369.

[5] J. Kollár, "Nonrational covers of $\mathbb{C P}^{m} \times \mathbb{C P}^{n}$ ", Explicit birational geometry of 3-folds, London Math. Soc. Lecture Note Ser., 281, Cambridge Univ. Press, Cambridge, 2000, $51-71$.

[6] А.В. Пухликов, "Бирациональные автоморфизмы трехмерных алгебраических многообразий с пучком поверхностей Дель Пеццо”, Изв. РАН. Сер. матем., 62:1 (1998), 123-164.

[7] J. Kollár, S. Mori (with the collaboration of C. H. Clemens and A. Corti), Birational geometry of algebraic varieties, Cambridge Tracts in Math., 134, Cambridge Univ. Press, Cambridge, 1998.

[8] A. V. Pukhlikov, "Essentials of the method of maximal singularities", Explicit birational geometry of 3-folds, London Math. Soc. Lecture Note Ser., 281, Cambridge Univ. Press, Cambridge, 2000, 73-100.

[9] В.А. Исковских, Ю.А. Манин, "Трехмерные квартики и контрпримеры к проблеме Люрота", Матем. сб., 86:1 (1971), 140-166.

[10] А.В. Пухликов, "Бирациональные автоморфизмы трехмерной квартики с простейшей особенностью", Матем. сб., 135:4 (1988), 472-496.

[11] A. Corti, M. Mella, "Birational geometry of terminal quartic 3-folds. I", Amer. J. Math., 126:4 (2004), 739-761.

[12] И. А. Чельцов, "Бирационально жесткие многообразия Фано”, УМН, 60:5 (2005), $71-160$.

[13] А.А. Загорский, "О трехмерных конических расслоениях", Матем. заметки, 21:6 (1977), 745-758.

[14] M. Miyanishi, "Algebraic methods in the theory of algebraic threefolds - surrounding the works of Iskovskikh, Mori, and Sarkisov", Algebraic varieties and analytic varieties (Tokyo, 1981), Adv. Stud. Pure Math., 1, ed. S. Iitaka, North-Holland, Amsterdam, 1983, 69-99.

[15] В.Г. Саркисов, "Бирациональные автоморфизмы расслоений коник”, Изв. АН СССР. Сер. матем., 44:4 (1980), 918-945.

[16] В. А. Исковских, "К проблеме рациональных расслоений на коники", Матем. сб., 182:1 (1991), 114-121.

[17] В.А. Исковских, "О критерии рациональности для расслоений на коники", Матем. сб., 187:7 (1996), 75-92. 
[18] A. Corti, "Factoring birational maps of threefolds after Sarkisov", J. Algebraic Geom., 4:2 (1995), 223-254.

[19] В. А. Исковских, Лекции по трехмерным алгебраическим многообразиям. Многообразия Фано, Изд-во Моск. ун-та, М., 1988.

[20] Ю.И. Манин, Кубические формы: алгебра, геометрия, арифметика, Наука, М., 1972.

[21] F. Hidaka, K. Watanabe, "Normal Gorenstein surfaces with ample anti-canonical divisor", Tokyo J. Math., 4:2 (1981), 319-330.

[22] M. Reid, "Nonnormal del Pezzo surfaces", Publ. Res. Inst. Math. Sci., 30:5 (1994), 695-727.

[23] J.-L. Colliot-Thélène, "Arithmétique des variétés rationnelles et problèmes birationnels", Proceedings of the International Congress of Mathematicians (Berkeley, CA, 1986), Amer. Math. Soc., Providence, RI, 1987, 641-653.

[24] J. Kollár, Y. Miyaoka, S. Mori, "Rationally connected varieties", J. Algebraic Geom., 1:3 (1992), 429-448.

[25] В.А. Исковских, "Факторизация бирациональных отображений рациональных поверхностей с точки зрения теории Мори”, УМН, 51:4 (1996), 3-72.

[26] J. Kollár, Y. Miyaoka, S. Mori, "Rational connectedness and boundedness of Fano manifolds", J. Differential Geom., 36:3 (1992), 765-779.

[27] В. А. Алексеев, "Об условиях рациональности трехмерных многообразий с пучком поверхностей дель Пеццо степени 4”, Матем. заметки, 41:5 (1987), 724-730.

[28] К.А. Шрамов, "К вопросу рациональности неособых трехмерных многообразий с пучком поверхностей дель Пеццо степени 4", Матем. сб., 197:1 (2006), 133-144.

[29] М. М. Гриненко, “Бирациональные свойства пучков поверхностей дель Пеццо степеней 1 и 2", Матем. сб., 191:5 (2000), 17-38.

[30] М. М. Гриненко, "О расслоениях на поверхности дель Пеццо", Матем. заметки, 69:4 (2001), 550-565.

[31] М. М. Гриненко, "О послойных перестройках расслоений на поверхности дель Пеццо степени 2", УМН, 56:4 (2001), 145-146.

[32] А. В. Пухликов, "Послойные бирациональные соответствия", Матем. заметки, 68:1 (2000), 120-130.

[33] J. Park, "Birational maps of del Pezzo fibrations", J. Reine Angew. Math., 538 (2001), 213-221.

[34] K. Takeuchi, Weak Fano 3-folds with del Pezzo fibration of degree one, Preprint, Gifu Shotoku Gakuen University, Yanaizu, 1999.

[35] М. М. Гриненко, “О двойном конусе над поверхностью Веронезе”, Изв. РАН. Сер. матем., 67:3 (2003), 5-22.

[36] М. М. Гриненко, "Структуры Мори на трехмерном многообразии Фано индекса 2 и степени 1", Труды МИАН, 246 (2004), 116-141.

[37] С. И. Хашин, "Бирациональные автоморфизмы двойного конуса Веронезе размерности три", Вестник Моск. ун-та. Сер. 1. Матем., мех., 1984, № 1, 13-16.

[38] K. Takeuchi, Weak Fano 3-folds with del Pezzo fibration of degree two, Preprint, Gifu Shotoku Gakuen University, Yanaizu, 2001.

[39] М.М.Гриненко, "Бирациональные свойства пучков поверхностей дель Пеццо степеней 1 и 2. II", Матем. сб., 194:5 (2003), 31-60.

[40] M. Reid, "Minimal models of canonical 3-folds", Algebraic varieties and analytic varieties (Tokyo, 1981), Adv. Stud. Pure Math., North-Holland, Amsterdam, 1983, 131180.

[41] М. М. Гриненко, "Бирациональные автоморфизмы трехмерного двойного конуса", Матем. сб., 189:7 (1998), 37-52. 
[42] М. М. Гриненко, "Новые структуры Мори на двойном пространстве индекса 2", УMH, 59:3 (2004), 163-164.

[43] G. Brown, A. Corti, F. Zucconi, "Birational geometry of 3-fold Mori fibre spaces", The Fano Conference (Turin, Italy, 2002), Univ. Torino, Turin, 2004, 235-275.

[44] И.В. Соболев, "Бирациональные автоморфизмы одного класса многообразий, расслоенных на кубические поверхности", Изв. РАН. Сер. матем., 66:1 (2002), 203-224.

[45] И. А. Чельцов, "Метод вырождения и нерациональность трехмерных многообразий с пучком поверхностей дель Пеццо", УМН, 59:4 (2004), 203-204.

[46] M. Reid, "Chapters on algebraic surfaces", Complex algebraic geometry, Lectures of a summer program (Park City, UT, 1993), IAS/Park City Math. Ser., 3, ed. J. Kollár, Amer. Math. Soc., Providence, RI, 1997, 5-159.

[47] В.В. Шокуров, "Многообразия Прима: теория и приложения", Изв. АН СССР. Сер. матем., 47:4 (1983), 785-855.

[48] T. Matsusaka, "Algebraic deformations of polarized varieties", Nagoya Math. J., 31 (1968), 185-245; corrections: ibid., 33, 1968, 137.

М. М. Гриненко (M. M. Grinenko)

Математический институт им. В. А. Стеклова РАН

Поступила в редакцию

E-mail: grin@mi.ras.ru 